\title{
FGT Old-Age Poverty Measures and the Mortality Paradox: Theory and Evidence*
}

\author{
Mathieu Lefèbvre ${ }^{\dagger}$ Pierre Pestieau ${ }^{\ddagger}$ Gregory Ponthiere ${ }^{\S}$
}

August 17, 2016

\begin{abstract}
Income-differentiated mortality, by reducing the share of poor persons in the population, leads to the "Mortality Paradox": the worse the survival conditions of the poor are, the lower the measured poverty is. We show that FGT measures (Foster Greer Thorbecke 1984) are, in general, not robust to variations in survival conditions. Then, following Kanbur and Mukherjee (2007), we propose to adjust FGT poverty measures by extending the income profiles of the prematurely dead, and we identify the condition under which so-adjusted FGT measures are robust to mortality changes. Finally, we show, on the basis of data on old-age poverty in 11 European economies (2007), that the effect of extending income profiles of the prematurely dead on poverty measurement varies with: (1) the fictitious income assigned to the prematurely dead; (2) the degree of poverty aversion; (3) the shape of the (unadjusted) income distribution; (4) the strength of the income/mortality relationship.

Keywords: income-differentiated mortality, FGT poverty measures.

JEL classification code: I32.
\end{abstract}

${ }^{*}$ The authors would like to thank Marion Leturcq, François Maniquet, Ramses Abul Naga, Yukihiro Nishimura, Daniel Szpiro, Alain Trannoy, Verity Watson, two anonymous referees, as well as participants of seminars at BETA (Strasbourg), LAGV (Aix-en-Provence), ERUDITE (Créteil) and the EEA Conference (Mannheim), for their comments and suggestions on this paper.

$\dagger$ University of Strasbourg, BETA.

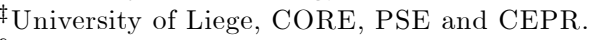

$\S$ University Paris East (ERUDITE), Paris School of Economics and Institut universitaire de France. Address: Ecole Normale Supérieure, 48 bd Jourdan, 75014 Paris, France. E-mail: gregory.ponthiere@ens.fr 


\section{Introduction}

In the recent decades, a voluminous empirical literature has emphasized that mortality risks are negatively correlated with income. ${ }^{1}$ Lower incomes are statistically related with higher mortality risks. The relationship between income and life expectancy is increasing, but non-linear, and exhibits a stronger slope at low income levels (Backlund et al 1999).

Income-differentiated mortality raises serious problems for poverty measurement. Clearly, if low-income individuals tend to face higher mortality risks than high-income individuals, standard poverty measures capture not only the true poverty, but, also, the selection induced by income-differentiated mortality. Indeed, if poor persons tend to die, on average, earlier than non-poor persons, it follows that there exist some "missing poor" persons at the old age, to use an expression close to Sen's (1998) "missing women".

The interferences or noise induced by income-differentiated mortality lead to what can be called the "Mortality Paradox": the worse the survival conditions of the poor are, the lower the measured poverty is. The Mortality Paradox is not caused by mortality per se, but by the correlation between income and mortality risks. That correlation, by creating a selection bias, introduces some interferences or noise in the measurement of poverty.

Note that, when facing selection biases due to income-differentiated mortality, adding another dimension, such as life expectancy, and shifting from one-dimensional poverty measures to two-dimensional poverty measures is not sufficient to avoid measurement problems. Indeed, multidimensional poverty indicators (even those taking life expectancy into account) compute poverty measures for a given population, and, as such, are also subject to the selection biases induced by income-differentiated mortality. Thus the correlation between income and mortality risks raises a general problem for the measurement of poverty, whatever one uses one-dimensional or multidimensional measures.

At first glance, the selection bias induced by income-differentiated mortality seems to lead to an underestimation of the poverty phenomenon. To illustrate this, take standard headcount poverty measures. If low-income individuals die earlier than non-poor individuals, those "missing poor" are not counted as poor. Assuming that income mobility is negligible, those poor individuals would have been counted as poor if they had faced the same survival conditions as the non-poor. Therefore headcount measures underestimate the extent of poverty.

However, once we consider other poverty measures, which are sensitive to the income distribution, the above rationale may not hold any more. Take, for instance, the class of poverty indicators known as the FGT measures (Foster Greer Thorbecke 1984). FGT measures are a parametric family of poverty measures where the parameter is an indicator of aversion to poverty. When that parameter equals 0 , the poverty measure collapses to a simple headcount ratio, but when that parameter is strictly positive, the poverty measure satisfies the Monotonicity Axiom (i.e. a reduction in the income of the poor must increase the poverty measure ceteris paribus). Moreover, if that parameter strictly exceeds 1, the poverty measure satisfies the Transfer Axiom (i.e. a pure transfer of income from a poor to someone richer must increase the poverty measure ceteris

\footnotetext{
${ }^{1}$ See, among others, Duleep (1986), Deaton and Paxson (1998), Backlund et al (1999), Deaton (2003), Jusot (2003), Duggan et al (2007) and Salm (2007). On the contrary, Snyder and Evans (2006) find the opposite correlation.
} 
paribus). Distribution-sensitive poverty measures such as FGT measures may not necessarily decrease when the survival conditions of some poor are worsened. The measured poverty index may either go up or down, depending on the overall effect of that rise of mortality on the income distribution.

The goal of this paper is to examine how income-differentiated mortality affects FGT poverty measures, and, in particular, whether income-differentiated mortality leads FGT measures to over- or underestimate the extent of poverty. For that purpose, we develop a simple theoretical model with income mobility and income-differentiated mortality, and study the behavior of FGT poverty measures in that framework. We pay a particular attention to the following questions. Are FGT measures subject to the Mortality Paradox? If yes, are all subclasses of FGT measures equally subject to that selection bias? How can we adjust FGT measures in order either to neutralize the effects of premature deaths, or, alternatively, to count premature deaths as a part of the poverty phenomenon to be measured?

The last question matters, since there are two possible attitudes regarding the treatment of premature deaths within measures of poverty. On the one hand, one may argue that adjusted poverty measures should not be affected by variations in mortality, and should thus be made robust with respect to variations in survival conditions. From that perspective, the construction of poverty measures should treat premature deaths as a kind of neutral thing, which does not affect the value taken by poverty measures. On the other hand, one may, following Sen (1998), consider that a premature death is itself a major source of deprivation, and that poverty measures should count premature deaths as a part of the poverty phenomenon to be measured. From that alternative perspective, poverty measures should not treat premature deaths as something neutral. Those two attitudes invite, as we shall see, different kinds of adjustment of poverty measures.

In order to examine those issues, we will proceed in three stages. In a first stage, we examine whether FGT measures of old-age poverty are robust or not to changes in survival conditions, and we identify particular income mobility processes under which those measures satisfy that robustness requirement. In a second stage, we propose, following the recent works by Kanbur and Mukherjee (2007) and Lefèbvre et al (2013), to construct adjusted FGT poverty measures by extending, through a fictitious income, the lifetime income profiles of the prematurely dead individuals, in such a way as to take those "missing poor" into account in the measurement of poverty. We show that there exist various ways to assign those fictitious incomes, each way reflecting how the evaluator wants the poverty measure to take premature deaths into account. We also show that, among those possible adjustments, one option is to make adjusted FGT measures robust to mortality changes by parametrizing the assignment of fictitious incomes in such a way as to fit the income mobility process conditional on survival. Finally, the behavior of FGT measures is illustrated empirically on the basis of old-age poverty data for 11 European countries for 2007.

Anticipating on our results, we first show that standard FGT measures of old-age poverty are not, in general, robust to changes in survival conditions. We also show that the invariance of FGT measures to changes in survival conditions holds when we restrict ourselves to a particular family of income mobility processes under which members of all income groups face the same expected extent of poverty in case of survival to the old age. That assumption being quite 
restrictive, we then propose to adjust FGT measures by extending the lifetime income profiles of the prematurely dead, and we identify the condition under which such an extension makes FGT measures robust to variations in mortality risk. Finally, the empirical application to Europe reveals that the effect of extending income profiles of the prematurely dead on poverty measurement varies significantly with: (1) the fictitious income assigned to the prematurely dead; (2) the degree of poverty aversion; (3) the shape of the (unadjusted) income distribution; (4) the strength of the income/mortality relationship.

In the light of those results, the present paper complements the existing literature on the measurement of poverty under income-differentiated mortality in two main ways. Firstly, the present paper extends the theoretical papers by Kanbur and Mukherjee (2007) and Lefèbvre et al (2013) by deriving, for the particular class of FGT poverty measures, formal conditions under which those measures are robust to changes in survival conditions, as well as conditions under which the extension of lifetime income profiles of the prematurely dead proposed in those two papers can make poverty measures robust to mortality changes. Secondly, we also provide, in the present paper, an empirical exploration of how the effects of extending the income profiles of the prematurely dead on poverty measurement vary across different adjustment techniques, across different degrees of poverty aversion, and across different countries. That empirical exploration, by showing how the treatment of the prematurely dead affects poverty measurement, suggests that the theoretical discussions in Kanbur and Mukherjee (2007) and Lefèbvre et al (2013) concern a general problem for poverty measurement, whose size varies with the degree of poverty aversion.

Finally, it should be stressed that our paper also complements the recent literature on the measurement of poverty along the life cycle (see Foster 2009, Bossert et al 2011, Hoy and Zheng, 2011, Dutta et al 2013). Those papers paid a particular attention to the question of how one can aggregate snapshot poverty measures into an aggregate measure of lifetime poverty. ${ }^{2}$ Although our study concentrates only on snapshot poverty measures, it nonetheless complements the literature on lifetime poverty, since the problem that we consider in this paper is also relevant for measures of lifetime poverty. Indeed, when comparing lives of different lengths, measures of lifetime poverty face similar difficulties regarding the treatment of the prematurely dead. Thus our study of the assignment of fictitious incomes to the prematurely dead could also be used to make lives of unequal durations comparable in the perspective of building an index of lifetime poverty (rather than the snapshot measure considered in this paper). Thus the problem discussed here is quite general, and applies also to multidimensional measures, including lifetime poverty measures.

The rest of the paper is organized as follows. Section 2 presents the model. Section 3 studies the robustness of FGT measures to changes in survival conditions. Section 4 proposes to extend income profiles of the prematurely dead, in such a way as to make adjusted FGT measures non-decreasing when the survival conditions of the poor worsen. Section 5 uses data on old-age poverty in 11 European countries to compare, across countries and gender, the effect of extending the lifetime income profiles of the prematurely dead on old-age poverty measurement. Section 6 concludes. The Appendix collects all proofs.

\footnotetext{
${ }^{2}$ From that perspective, a particular question concerns how episodes of chronic poverty should be counted within measures of lifetime poverty.
} 


\section{The model}

We consider a two-period economy, where a cohort, of size $N \in \mathbb{N}$, lives the young age (first period) for sure, whereas only some fraction of the population will enjoy the old age (second period). We assume that there exists a perfect rank correlation between, on the one hand, income levels at the young age, and, on the other hand, survival chances to the old age. Hence a higher income when being young leads to a higher probability of survival to the old age. That model is in line with the empirical evidence suggesting that income and longevity are positively correlated. ${ }^{3}$

The economy takes the following form. There exists a finite number $K \in \mathbb{N}$ of possible income levels $(K>1), y_{1}, \ldots, y_{K} \in \mathbb{R}_{+}$. Income levels are indexed in an increasing order. Among the $K$ income levels $y_{1}, \ldots, y_{K}$, there exists one particular (intermediate) income level, denoted by $y_{P}$, which is the poverty line. We thus have:

$$
y_{1}<\ldots<y_{P}<\ldots<y_{K}
$$

The poverty line $y_{P}$ is supposed to be invariant. All income classes $y_{k}$ from $k=1$ to $k=P-1$ are assumed to be in poverty, whereas all income classes $y_{k}$ from $k=P$ to $k=K$ are assumed not to be in poverty.

Throughout the paper, we will denote a $K \times 1$ vector of possible income levels (including the fixed poverty line $y_{P}$ ) by $\mathbf{y}$, and denote by $\mathbf{Y}$ the set of all possible vectors $\mathbf{y}$. Given that $\mathbf{y}$ are vectors whose entries are strictly increasing, the set of all income vectors is defined as: $\mathbf{Y}=\left\{\mathbf{y} \in \mathbb{R}_{+}^{K} \mid y_{1}<\ldots<y_{P}<\ldots<y_{K}\right\} .{ }^{4}$

The number of young individuals with income $y_{k}$ is denoted by $n_{k} \in \mathbb{N} .{ }^{5}$ We denote by $\mathbf{n}$ the vector of size $K$, whose entries are $n_{k}$ for $k=1, \ldots, K$. Throughout the paper, we will denote by $\mathbf{N}=\mathbb{N}^{K}$ the set of all vectors $\mathbf{n}$.

Among each income group, some members die before reaching the old age, whereas others reach the old age. Let us denote by $s_{k}$ the number of survivors within an income group $y_{k}$. We suppose, without loss of generality, that the number of surviving members of an income group $y_{k}$ is a natural number, i.e. $s_{k} \in \mathbb{N}$, which is assumed to be strictly lower than $n_{k}: s_{k}<n_{k}$. The proportion of survivor in an income group $y_{k}$ is denoted by $\pi_{k}$, where:

$$
\pi_{k}=\frac{s_{k}}{n_{k}}
$$

Given that both $n_{k} \in \mathbb{N}$ and $s_{k} \in \mathbb{N}$, we have $\pi_{k} \in \mathbb{Q}_{+}$. Given $s_{k}<n_{k}$, we necessarily have $0<\pi_{k}<1$.

Incomes at the young age and survival probabilities towards the old age are assumed to have the same ranking over the different classes $k=1, \ldots, K$. Thus, income-specific survival probabilities to the old age $\pi_{k}$ for income groups $k=1, \ldots, K$ are ranked in an increasing order:

$$
\pi_{1}<\ldots<\pi_{P}<\ldots<\pi_{K}
$$

\footnotetext{
${ }^{3}$ See Duleep (1986), Deaton and Paxson (1998), Jusot (2004) and Salm (2007)

${ }^{4}$ This definition of $\mathbf{Y}$ implies that, among all income vectors considered throughout the paper, the income level corresponding to the poverty line $y_{P}$ is always present as an entry of such vectors.

${ }^{5}$ We have: $\sum_{k=1}^{K} n_{k}=N$.
} 
where $\pi_{P}$ is the proportion of survivors in the income group $y_{P}$ coinciding with the poverty line. We denote by $\boldsymbol{\pi}$ the vector of size $K$ whose entries are the income-specific survival probabilities $\pi_{k}$. In the rest of this paper, we will denote by $\boldsymbol{\Pi}$ the set of all possible vectors $\boldsymbol{\pi}$. Given that $\boldsymbol{\pi}$ are vectors whose entries are strictly increasing, we can define the set of all possible vectors $\boldsymbol{\pi}$ as $\boldsymbol{\Pi}=\left\{\boldsymbol{\pi} \in \mathbb{Q}_{+}^{\mathbf{K}} \mid \pi_{1}<\ldots<\pi_{P}<\ldots<\pi_{K}\right\}$.

The number of surviving old individuals with income $y_{i}$ is denoted by $m_{i} \in$ $\mathbb{N}$. We denote by $\mathbf{m}$ the vector of size $K$, whose entries are $m_{k}$ for $k=1, \ldots, K{ }^{6}$ Throughout the paper, we will denote by $\mathbf{M}=\mathbb{N}^{K}$ the set of all vectors $\mathbf{m}$.

The income mobility process depends both on the income-specific survival rates, and on the chances to shift to different income levels in case of survival. The probability that a young agent with income $y_{i}$ enjoys, in case of survival, an income $y_{k}$ at the old age, denoted by $\lambda_{i k}$, can be defined as:

$$
\lambda_{i k}=\frac{m_{i k}}{s_{i}}
$$

where $m_{i k} \in \mathbb{N}$ is the number of surviving old individuals who enjoy income $y_{k}$ at the old age, and who enjoyed income $y_{i}$ when being young. ${ }^{7}$ Given that $m_{i k} \in \mathbb{N}$ and $s_{i} \in \mathbb{N}$, we have $\lambda_{i k} \in \mathbb{Q}_{+}$. Given $m_{i k} \leq s_{i}$, we necessarily have $0<\lambda_{i k} \leq 1$. Note also that we necessarily have, for a given income $y_{i}$ enjoyed at the young age, that:

$$
\sum_{k=1}^{K} \lambda_{i k}=\sum_{k=1}^{K} \frac{m_{i k}}{s_{i}}=1
$$

In the following, we denote by $\boldsymbol{\Lambda}$ the $K \times K$ matrix that describes the "pure" income mobility process, that is, the income mobility process conditionally on survival to the old age:

$$
\boldsymbol{\Lambda} \equiv\left(\begin{array}{cccc}
\lambda_{11} & \lambda_{12} & \ldots & \lambda_{1 K} \\
\lambda_{21} & \lambda_{22} & \ldots & \lambda_{2 K} \\
\ldots & \ldots & \ldots & \ldots \\
\lambda_{K 1} & \lambda_{K 2} & \ldots & \lambda_{K K}
\end{array}\right)
$$

The set of all matrixes $\boldsymbol{\Lambda}$ is $\mathbf{L}=\left\{\boldsymbol{\Lambda} \in \mathbb{Q}_{+}^{\mathbf{K} \times \mathbf{K}} \mid \sum_{k=1}^{K} \lambda_{i k}=1 \forall i=1, \ldots, K\right\}$.

In the absence of income-differentiated mortality, the matrix $\boldsymbol{\Lambda}$ would include all relevant information about the dynamics of income distribution over time. However, given the existence of income-differentiated mortality, the matrix $\boldsymbol{\Lambda}$ does not provide a complete description of the income mobility process. The number of old individuals with, for instance, income $y_{k}$, denoted by $m_{k}$, is equal to the sum of all young individuals (with potentially any income level) who (1) reached the old age and (2) turned out to move from their income level at the young age to the income group $k$ at the old age, that is:

$$
m_{k}=\sum_{i=1}^{K} \pi_{i} n_{i} \lambda_{i k}
$$

${ }^{6}$ We have: $\sum_{k=1}^{K} m_{k}=\sum_{k=1}^{K} \pi_{k} n_{k}$.

${ }^{7}$ We obviously have, for any income $y_{i}$ enjoyed at the young age, that: $\sum_{k=1}^{K} m_{i k}=s_{i}$. 
If there was no mortality $\left(\pi_{i}=1 \forall i\right)$ and no income mobility $\left(\lambda_{i i}=1 \forall i\right)$, we would have $m_{k}=n_{k}$. However, given the existence of mortality and income mobility, it is most likely that $m_{k} \neq n_{k}$.

Actually, given income-differentiated mortality, the income mobility process can be described by means of a $K \times K$ transition matrix, denoted by $\Omega$, which describes how the income distribution at the young age determines the income distribution at the old age:

$$
\mathbf{m}=\mathbf{\Omega}^{\prime} \mathbf{n}
$$

where the $\boldsymbol{\Omega}$ matrix is defined as:

$$
\boldsymbol{\Omega} \equiv\left(\begin{array}{cccc}
\pi_{1} \lambda_{11} & \pi_{1} \lambda_{12} & \ldots & \pi_{1} \lambda_{1 K} \\
\pi_{2} \lambda_{21} & \pi_{2} \lambda_{22} & \ldots & \pi_{2} \lambda_{2 K} \\
\ldots & \ldots & \ldots & \ldots \\
\pi_{K} \lambda_{K 1} & \pi_{K} \lambda_{K 2} & \ldots & \pi_{K} \lambda_{K K}
\end{array}\right)
$$

The $\boldsymbol{\Omega}$ matrix fully describes the trajectories of individuals in our economy. The lifecycle trajectory depends on survival probabilities and on income transition probabilities, which are correlated in terms of rank. We can easily rewrite the matrix $\boldsymbol{\Omega}$ as the Hadamard product (i.e. the entrywise product) of the following two matrices: ${ }^{8}$

$$
\boldsymbol{\Omega}=\boldsymbol{\Lambda} \circ \boldsymbol{\Xi}
$$

where the matrix $\boldsymbol{\Xi}$ is:

$$
\boldsymbol{\Xi} \equiv\left(\begin{array}{cccc}
\pi_{1} & \pi_{1} & \ldots & \pi_{1} \\
\pi_{2} & \pi_{2} & \ldots & \pi_{2} \\
\ldots & \ldots & \ldots & \ldots \\
\pi_{K} & \pi_{K} & \ldots & \pi_{K}
\end{array}\right)
$$

Given that $\mathbf{m}=\boldsymbol{\Omega}^{\prime} \mathbf{n}$, the income distribution at the old age $\mathbf{m}$ is, for a given initial income distribution $\mathbf{n}$, fully determined by the matrix $\boldsymbol{\Omega}$. Therefore the income distribution at the old age can be described in two alternative ways.

- The income distribution at the old age can be described by the collection:

$$
(\mathbf{y}, \mathbf{m})
$$

- The income distribution at the old age can also be described by the collection:

$$
(\mathbf{y}, \mathbf{n}, \boldsymbol{\pi}, \mathbf{\Lambda})
$$

The difference between the two definitions is that, while the definition $(\mathbf{y}, \mathbf{m})$ is static (since it only shows the sizes of the different income groups at the old age), the definition $(\mathbf{y}, \mathbf{n}, \boldsymbol{\pi}, \boldsymbol{\Lambda})$ is dynamic, since it tells us how the distribution of income at the old age $\mathbf{m}$ was achieved while starting from the income distribution at the young age $\mathbf{n}$. Given that dynamic nature, one can refer to $(\mathbf{y}, \mathbf{n}, \boldsymbol{\pi}, \boldsymbol{\Lambda})$ either as the income distribution at the old age, or as the income mobility process.

That second (dynamic) description of the income distribution at the old age is most relevant for the purpose of this study, and, hence, will be largely

\footnotetext{
${ }^{8}$ The symbol o refers to the Hadamard product.
} 
used throughout the rest of this paper. The reason lies in the fact that the definition of income distribution $(\mathbf{y}, \mathbf{n}, \boldsymbol{\pi}, \boldsymbol{\Lambda})$ allows us to study how the income distribution at the old age depends on the vector of income-specific survival probabilities.

\section{Robustness of FGT measures}

Let us now consider how to measure poverty in the economy under study. As this is well-known, there exist various families of poverty measures, each of these satisfying some particular properties or axioms. We will focus here on a particular family of poverty measures, which are called FGT poverty measures (see Foster et al 1984). Moreover, given that we are concerned here with the impact of premature death on the measurement of poverty at the old age, we will concentrate exclusively on FGT measures of old-age poverty.

Definition 1 Given an aversion to poverty $\alpha \geq 0$, and a poverty line $y_{P}$, the FGT poverty measure at the old age is a mapping $P_{\alpha}: \mathbf{Y} \times \mathbf{M} \rightarrow \mathbb{R}_{+}$:

$$
P_{\alpha}(\mathbf{y}, \mathbf{m})=\frac{1}{\sum_{j=1}^{K} m_{j}} \sum_{k=1}^{P-1} m_{k}\left[\frac{y_{P}-y_{k}}{y_{P}}\right]^{\alpha}
$$

As stressed in Foster et al (1984), the parameter $\alpha$ can be interpreted as an indicator of aversion to poverty. When $\alpha=0$, the poverty index is a headcount ratio, which, as such, is not reactive to income reductions of the poor. However, once $\alpha>0$, income reductions of the poor increase, ceteris paribus, the measured poverty, in line with the Monotonicity Axiom. Moreover, when $\alpha>1$, transfers of income from a poor to a richer person raise, ceteris paribus, the level taken by the poverty measure, in line with the Transfer Axiom.

Given that this paper is concerned with selection biases induced by incomedifferentiated mortality, it is useful to provide another definition of the FGT old-age poverty measure, that does not rely on the domain $\mathbf{Y} \times \mathbf{M}$, but, rather, that relies on the domain $\mathbf{Y} \times \mathbf{N} \times \mathbf{\Pi} \times \mathbf{L}$, in such a way as to take history into account. Indeed, as stated above, the domain $\mathbf{Y} \times \mathbf{N} \times \mathbf{\Pi} \times \mathbf{L}$ allows us to take into account how the distribution of income at the old age $\mathbf{m}$ was achieved while starting from the income distribution at the young age $\mathbf{n}$, which is most relevant for considering selection issues.

Definition 2 Given an aversion to poverty $\alpha \geq 0$, and a poverty line $y_{P}$, the $F G T$ poverty measure at the old age can also be defined as a mapping $\bar{P}_{\alpha}$ : $\mathbf{Y} \times \mathbf{N} \times \mathbf{\Pi} \times \mathbf{L} \rightarrow \mathbb{R}_{+}:$

$$
\bar{P}_{\alpha}(\mathbf{y}, \mathbf{n}, \boldsymbol{\pi}, \boldsymbol{\Lambda})=\frac{1}{\sum_{k=1}^{K} \sum_{i=1}^{K} \pi_{i} n_{i} \lambda_{i k}} \sum_{k=1}^{P-1} \sum_{i=1}^{K} \pi_{i} n_{i} \lambda_{i k}\left[\frac{y_{P}-y_{k}}{y_{P}}\right]^{\alpha}
$$

By highlighting the role of survival conditions, that alternative definition of the old-age FGT poverty measure is most adequate for exploring the robustness of poverty measures to changes in survival conditions. Indeed, this definition, by relying on the domain $\mathbf{Y} \times \mathbf{N} \times \mathbf{\Pi} \times \mathbf{L}$, allows us to examine how the measured poverty varies when there is a change in survival conditions, that is, a change in the vector $\pi$. 
In order to study the sensitivity of FGT poverty measures to changes in survival conditions, we need first to define formally what we mean by a poverty measure that is robust to changes in mortality. The following property, entitled Robustness to Mortality Changes (RMC), captures some idea of robustness to changes in survival conditions.

Definition 3 A poverty measure $\bar{P}_{\alpha}$ satisfies Robustness to Mortality Changes $(R M C)$ if and only if, for all $(\mathbf{y}, \mathbf{n}, \boldsymbol{\pi}, \boldsymbol{\Lambda}) \in \mathbf{Y} \times \mathbf{N} \times \mathbf{\Pi} \times \mathbf{L}$ and for all $\boldsymbol{\pi}^{\prime} \in$ $\boldsymbol{\Pi}$, if there exists $k \in\{1, \ldots, K\}$ with $\pi_{k} \neq \pi_{k}^{\prime}$ and $\pi_{j}=\pi_{j}^{\prime}$ for other $j \in$ $\{1, \ldots, K\} \backslash\{k\}$, then

$$
\bar{P}_{\alpha}(\mathbf{y}, \mathbf{n}, \boldsymbol{\pi}, \boldsymbol{\Lambda})=\bar{P}_{\alpha}\left(\mathbf{y}, \mathbf{n}, \boldsymbol{\pi}^{\prime}, \mathbf{\Lambda}\right)
$$

The RMC property states that poverty values do not depend on the survival rates. In other words, the RMC property states that the level taken by the poverty measure should be left invariant to any variation in survival rates, whatever the income group considered. That requirement, if satisfied, makes poverty measures immunized against the noise due to income-differentiated mortality. Hence, one can regard the RMC property as a condition guaranteeing that the poverty measure escapes from the Mortality Paradox, and thus does not exhibit lower values when the survival conditions of the poor are worsened.

Regarding the desirability of the RMC property, it should be stressed here that this issue is open to debates, and depends on what one would like the poverty measure to capture. Clearly, if one believes that poverty measures should not be sensitive to the fact that some individuals die earlier than others, then the RMC property is desirable, since it makes the poverty measure fully robust to variations in survival conditions, and, hence, immunized against selection effects. However, if one, following Sen (1998), considers that the mere fact of dying prematurely is a major source of deprivation in itself, which should affect the levels of poverty indicators in the sense that earlier deaths should lead to higher measured poverty, then RMC is no longer a desirable property. We will consider that issue in the next section.

At this stage, it is important to stress that the RMC property constitutes a quite strong invariance requirement, since it requires the invariance of the value taken by old-age poverty measure to hold whatever the income mobility process $(\mathbf{y}, \mathbf{n}, \boldsymbol{\pi}, \boldsymbol{\Lambda})$ is. Given the various forms that $(\mathbf{y}, \mathbf{n}, \boldsymbol{\pi}, \boldsymbol{\Lambda})$ can take, the RMC condition imposes, in fact, a very strong requirement on poverty measures.

In the light of this, it does not come as a surprise that the FGT poverty measure does not satisfy RMC.

Proposition 1 The FGT measure $\bar{P}_{\alpha}$ does not satisfy $R M C$.

The reason why FGT poverty measures violate RMC lies in the strength of that robustness condition: RMC does not require the values taken by $\bar{P}_{\alpha}(\mathbf{y}, \mathbf{n}, \boldsymbol{\pi}, \boldsymbol{\Lambda})$ to be invariant to some changes in survival probabilities, but to any of them. To put it differently, it could be the case that some variation in a survival probability leaves the value of the poverty measure unchanged. However, requiring that any change in the survival conditions leaves the measured poverty unchanged is a quite demanding requirement.

The strength of the RMC requirement comes also from the absence of restrictions on the form of the income mobility process $(\mathbf{y}, \mathbf{n}, \boldsymbol{\pi}, \boldsymbol{\Lambda})$. Indeed, there 
could exist some subclasses of income mobility processes $\{\mathbf{y}, \mathbf{n}, \boldsymbol{\pi}, \boldsymbol{\Lambda}\}$ such that the FGT measure $\bar{P}_{\alpha}$ satisfies RMC. Proposition 2 states the general restrictions on the space $\mathbf{Y} \times \mathbf{N} \times \mathbf{\Pi} \times \mathbf{L}$ under which FGT measures satisfy RMC.

Proposition $\mathbf{2}$ Let $\mathbf{L}^{\prime}$ be the subset of $\mathbf{L}$ and $\mathbf{Y}^{\prime}$ be the subset of $\mathbf{Y}$ such that, for all $\mathbf{\Lambda} \in \mathbf{L}^{\prime}$, and for all $\mathbf{y} \in \mathbf{Y}^{\prime}$, we have, for all $i, j \in\{1, \ldots, K\}$ :

$$
\lambda_{i 1} z_{1}+\lambda_{i 2} z_{2}+\ldots+\lambda_{i P-1} z_{P-1}=\lambda_{j 1} z_{1}+\lambda_{j 2} z_{2}+\ldots+\lambda_{j P-1} z_{P-1}
$$

where $z_{k} \equiv\left(\frac{y_{P}-y_{k}}{y_{P}}\right)^{\alpha}$. Then the restriction of the FGT measure $\bar{P}_{\alpha}$ to $\mathbf{Y}^{\prime} \times \mathbf{N} \times \mathbf{\Pi} \times \mathbf{L}^{\prime}$ satisfies $R M C$.

The intuition behind Proposition 2, which states a sufficient condition (restrictions on the space $\mathbf{Y} \times \mathbf{N} \times \boldsymbol{\Pi} \times \mathbf{L}$ ) to achieve RMC, goes as follows. When the members of all income groups face the same expected extent of poverty in case of survival to the old age, changes in survival conditions cannot affect the measured extent of poverty, since the measured poverty is then independent from survival conditions.

To illustrate Proposition 2, let us consider a simple example with 3 income levels satisfying $y_{1}<y_{2}<y_{3}$ with $y_{P}=y_{2}$. Let us also suppose that $\boldsymbol{\Lambda}$ and $\mathbf{y}$ are such that we have, in line with Proposition 2:

$$
\lambda_{11} z_{1}=\lambda_{21} z_{1}=\lambda_{31} z_{1}=z
$$

The old-age poverty measure gives here a value:

$$
\bar{P}_{\alpha}(\mathbf{y}, \mathbf{n}, \boldsymbol{\pi}, \boldsymbol{\Lambda})=\frac{n_{1} \pi_{1} z+n_{2} \pi_{2} z+n_{3} \pi_{3} z}{n_{1} \pi_{1}+n_{2} \pi_{2}+n_{3} \pi_{3}}=z
$$

Hence the old-age poverty measure gives a value equal to $z$, whatever the incomespecific survival probabilities are, and is thus also invariant to any change in survival conditions.

A simple corollary of Proposition 2 can be derived for FGT old age poverty measures with a degree of poverty aversion equal to 0 (i.e. when $\alpha=0$ ).

Corollary $\mathbf{1}$ Let $\mathbf{L}^{\prime}$ be the subset of $\mathbf{L}$ such that, for all $\mathbf{\Lambda} \in \mathbf{L}^{\prime}$,

$$
\lambda_{i 1}+\lambda_{i 2}+\ldots+\lambda_{i P-1}=\lambda_{j 1}+\lambda_{j 2}+\ldots+\lambda_{j P-1}
$$

for all $i, j \in\{1, \ldots, K\}$. Then the restriction of the FGT measure $\bar{P}_{0}$ to $\mathbf{Y} \times \mathbf{N} \times \mathbf{\Pi} \times \mathbf{L}^{\prime}$ satisfies $R M C$.

Corollary 1 suggests that, if one focuses on a particular subclass of income mobility processes $(\mathbf{y}, \mathbf{n}, \boldsymbol{\pi}, \boldsymbol{\Lambda})$, FGT measures with zero poverty aversion - that is, headcount poverty measures - do satisfy RMC. The specificity of the income mobility processes consists of similar probabilities of falling into poverty in case of survival to the old age whatever the initial income level is.

It is straightforward to see that Corollary 1 is a special case of Proposition 2 for FGT poverty measures exhibiting a zero degree of aversion to poverty. Indeed, under headcount measures, we have $z_{i}=1$ for all income groups, so that the condition of Proposition 2 vanishes to the condition of Corollary 1, that is, the equality of the probabilities to fall into poverty in case of survival to the old age. 
Let us illustrate the result of Corollary 1 with the following economy, with two possible income levels satisfying $y_{1}<y_{2}$. The poverty line is fixed to the level $y_{2}$, so that the population is partitioned between poor (i.e. with income $y_{1}$ ) and non-poor (i.e. with income $y_{2}$ ). The condition mentioned in Corollary 1 consists of assuming that the matrix $\boldsymbol{\Lambda}$ takes the following form:

$$
\mathbf{\Lambda}=\left(\begin{array}{cc}
a & 1-a \\
a & 1-a
\end{array}\right)
$$

It is clear that, whatever the survival conditions faced by the two income groups are (i.e. $\pi_{1}$ and $\pi_{2}$ ), and whatever the initial income distribution is, the proportion of poor persons at the old age is necessarily equal to $a$. Thus equal probabilities to fall into poverty in case of survival to the old age guarantee that the headcount poverty measure is robust to mortality changes. ${ }^{9}$

To illustrate Corollary 1 further, let us consider the following matrix $\boldsymbol{\Lambda}$, with three possible income levels satisfying $y_{1}<y_{2}<y_{3}$ :

$$
\boldsymbol{\Lambda}=\left(\begin{array}{ccc}
a & b & 1-a-b \\
a & c & 1-a-c \\
a & d & 1-a-d
\end{array}\right)
$$

When $y_{P}=y_{2}$, it is easy to see that the proportion of poor persons at the old age is necessarily equal to $a$, whatever the survival rates and initial income distributions are. However, if $y_{P}=y_{3}$, this is no longer the case. Under that alternative poverty line, the RMC property requires necessarily $b=c=d$, in such a way as to equalize the income-specific probabilities to fall into poverty across income groups.

Taken together, Propositions 2 and Corollary 1 show that there exist some subclasses of economies for which the FGT poverty measure does satisfy the RMC requirement. However, it should be stressed here that the restrictions imposed on the income mobility process $(\mathbf{y}, \mathbf{n}, \boldsymbol{\pi}, \boldsymbol{\Lambda})$ so as to achieve RMC are extremely strong. Actually, one expects that, in the real world, members of different income groups are not characterized by the same expected extent of poverty at the old age, unlike what is required in Proposition 2. Indeed, poor persons at the young age are more likely to face higher expected extent of poverty at the old age than persons who are not poor at the young age.

Hence, except if the particular restrictions imposed in Proposition 2 on the space $\mathbf{Y} \times \mathbf{N} \times \mathbf{\Pi} \times \mathbf{L}$ hold, FGT poverty measures do not satisfy the RMC property. Thus FGT old-age poverty measures are not, except under those restrictive conditions, robust to variations in survival conditions. As such, these are likely to be subject to the Mortality Paradox, in the sense that a worsening of the survival conditions of the poor may reduce the measured poverty.

Given that the FGT poverty measure does not satisfy RMC, one may wonder what happens to the value of the poverty measure when there is a change in a single survival rate. Actually, when a survival rate $\pi_{j}$ with $1 \leq j \leq K$ decreases

\footnotetext{
${ }^{9}$ A particular consequence of Corollary 1 concerns economies with no income mobility, that is, for which the $\boldsymbol{\Lambda}$ matrix is a diagonal matrix. Indeed, if $\boldsymbol{\Lambda}$ is a diagonal matrix, the property mentioned in Corollary 1 cannot be satisfied. Hence RMC must be violated.
} 
to $\pi_{j}^{\prime}<\pi_{j}$, we have:

$$
\begin{aligned}
& \bar{P}_{\alpha}(\mathbf{y}, \mathbf{n}, \boldsymbol{\pi}, \boldsymbol{\Lambda}) \gtrless \bar{P}_{\alpha}\left(\mathbf{y}, \mathbf{n}, \boldsymbol{\pi}^{\prime}, \boldsymbol{\Lambda}\right) \\
& \operatorname{iff} \frac{\sum_{i \neq j}^{K} \pi_{i} n_{i}\left(\sum_{k=1}^{P-1} \lambda_{i k}\left[\frac{y_{P}-y_{k}}{y_{P}}\right]^{\alpha}\right)+\pi_{j} n_{j}\left(\sum_{k=1}^{P-1} \lambda_{j k}\left[\frac{y_{P}-y_{k}}{y_{P}}\right]^{\alpha}\right)}{\sum_{i \neq j}^{K} \pi_{i} n_{i}+\pi_{j} n_{j}} \\
\gtrless & \frac{\sum_{i \neq j}^{K} \pi_{i} n_{i}\left(\sum_{k=1}^{P-1} \lambda_{i k}\left[\frac{y_{P}-y_{k}}{y_{P}}\right]^{\alpha}\right)+\pi_{j}^{\prime} n_{j}\left(\sum_{k=1}^{P-1} \lambda_{j k}\left[\frac{y_{P}-y_{k}}{y_{P}}\right]^{\alpha}\right)}{\sum_{i \neq j}^{K} \pi_{i} n_{i}+\pi_{j}^{\prime} n_{j}}
\end{aligned}
$$

A deterioration of the survival conditions faced by an income group $j$ leads to a fall or a rise in the extent of measured poverty, depending on the relative size of the second term of the numerator (i.e. the expected extent of poverty at the old age for income group $j$ ) with respect to the first term (i.e. the expected extent of poverty at the old age for all other income groups $i \neq j$ ). Thus, whether measured poverty will fall or grow depends on the expected extent of poverty that the prematurely dead of income group $j$ would have, in case of survival, suffered from, and on how large this expected extent of poverty is with respect to the expected extent of poverty among the rest of the population.

Clearly, if young individuals with income $y_{j}$ have little probability to fall into poverty at the old age, and thus would, in case of survival, face a small expected extent of poverty, the second term of the numerator is negligible, and it is easy to see that, given that $\pi_{j}^{\prime}<\pi_{j}$, the right-hand side (RHS) of the condition is likely to be larger than the left-hand side (LHS), implying that the level taken by the poverty measure increases when the survival conditions of income group $y_{j}$ deteriorate. On the contrary, if income group $j$ faces a large expected poverty, because of a large probability to fall into poverty in case of survival to the old age, then the second term of the numerator is large, which implies large effects from variations in $\pi_{j}$. In that case, it is likely that the LHS exceeds the RHS, so that a deterioration of the survival probability $\pi_{j}$ is likely to lead to a fall in the measured poverty.

In sum, a worsening of the survival conditions of some income group may either reduce or decrease the measured poverty, depending on whether the prematurely dead would have, in case of survival, suffered from an equal extent of poverty in comparison with the rest of the surviving population. When the deterioration of survival conditions concerns a group with a higher probability to be poor at the old age than the remaining surviving population, as well as having a larger income gap with respect to the poverty line, the worsening of survival conditions reduces the measured poverty. Alternatively, when gains in life expectancy only concern a high income group, with a low probability to become poor in case of survival, the measured poverty is reduced. Thus, there exist several ways in which changes in survival conditions affect the measured poverty, and disconnect it from the "true" poverty.

\section{Adjusting FGT measures}

As shown in the previous section, FGT old-age poverty measures are, in general, not robust to variations in survival conditions, and, hence, may be take either higher or lower levels when the survival chances of some income group vary. Such a lack of robustness is problematic for two different reasons. 
On the one hand, one may consider that poverty measures should, in general, satisfy RMC. Indeed, one can consider that income-differentiated mortality introduces interferences or noise in the measurement of old-age poverty, and that "good" poverty measures should be immunized against such interferences, and should count premature death as something "neutral" for poverty measurement. On the other hand, one may consider that poverty measures should not necessarily satisfy RMC, but should count premature death as a part of the poverty phenomenon to be measured, as argued by Sen (1998).

But whatever the precise motivation is, it is worth considering how one could adjust poverty measures, in such a way as to make these either robust to mortality changes, or, alternatively, to make these count premature death as a part of the poverty phenomenon to be measured.

For that purpose, we will follow here the approach proposed by Kanbur and Mukherjee (2007): the extension of the lifetime income profiles of the prematurely dead persons, in such a way as to count these when measuring the extent of the poverty phenomenon. ${ }^{10}$ The underlying intuition behind the adjustment proposed by Kanbur and Mukherjee (2007) consists of doing "as if" the prematurely dead individuals were still alive, in such a way as to make poverty measures robust to variations in survival conditions.

The extension of the lifetime income profiles of the prematurely dead persons is carried out by assigning a fictitious income to the prematurely dead. The assignment of a fictitious income to the prematurely dead can take various forms, depending on: (1) whether the assignment of fictitious incomes concerns all individuals or only the initially poor; (2) whether fictitious incomes exceed or are below the poverty line $y_{P}$. Those two features of the extension are captured by the $K \times K$ matrix $\Sigma$ :

$$
\boldsymbol{\Sigma} \equiv\left(\begin{array}{cccc}
\sigma_{11} & \ldots & \ldots & \sigma_{1 K} \\
\ldots & \ldots & \ldots & \ldots \\
\ldots & \ldots & \ldots & \ldots \\
\sigma_{K 1} & \ldots & \ldots & \sigma_{K K}
\end{array}\right)
$$

where $\sigma_{i j}$ is the probability, for an individual with income $y_{i}$ when being young, to have a fictitious income $y_{j}$ assigned to him if he dies prematurely. The set of all possible matrix $\boldsymbol{\Sigma}$ is denoted by $\mathbf{S}=\left\{\boldsymbol{\Sigma} \in \mathbb{Q}_{+}^{\mathbf{K} \times \mathbf{K}} \mid \sum_{k=1}^{K} \sigma_{i k}=1 \forall i=1, \ldots, K\right\}$.

The matrix $\boldsymbol{\Sigma}$ determines how prematurely dead persons are taken into account in the measurement of poverty. In other words, each particular treatment of the prematurely dead persons corresponds to a particular form of the matrix $\boldsymbol{\Sigma}$. Thus, the matrix $\boldsymbol{\Sigma}$ constitutes a part of the poverty measure itself, and not of the poverty phenomenon to be measured. This captures the judgements of the evaluator concerning the precise way in which he would like premature deaths to be taken into account when measuring poverty.

The extension of the lifetime income profiles of the prematurely dead through the matrix $\boldsymbol{\Sigma}$ leads to the following adjusted FGT poverty measures.

Definition 4 Given an aversion to poverty $\alpha \geq 0$, given a poverty line $y_{P}$ and given a $K \times K$ matrix $\boldsymbol{\Sigma}$ the entries $\sigma_{i j}$ of which are the probability, for

\footnotetext{
${ }^{10}$ Note, however, an important difference with respect to Kanbur and Mukherjee (2007): the extension of lifetime income profiles concerns here all prematurely dead persons, and not only the prematurely dead poor ones.
} 
an individual with income $y_{i}$ when being young, to have a fictitious income $y_{j}$ assigned to him when he is dead, the adjusted FGT poverty measure at the old age is a mapping $\hat{P}_{\alpha, \Sigma}: \mathbf{Y} \times \mathbf{N} \times \mathbf{\Pi} \times \mathbf{L} \rightarrow \mathbb{R}_{+}$:

$$
\hat{P}_{\alpha, \Sigma}(\mathbf{y}, \mathbf{n}, \boldsymbol{\pi}, \boldsymbol{\Lambda})=\frac{\left[\begin{array}{l}
\sum_{j=1}^{K} \pi_{j} n_{j}\left(\sum_{k=1}^{P-1} \lambda_{j k}\left[\frac{y_{P}-y_{k}}{y_{P}}\right]^{\alpha}\right) \\
+\sum_{j=1}^{K}\left(1-\pi_{j}\right) n_{j}\left(\sum_{k=1}^{P-1} \sigma_{j k}\left[\frac{y_{P}-y_{k}}{y_{P}}\right]^{\alpha}\right)
\end{array}\right]}{\sum_{j=1}^{K} n_{j}}
$$

The adjusted poverty measure $\hat{P}_{\alpha, \Sigma}(\mathbf{y}, \mathbf{n}, \boldsymbol{\pi}, \boldsymbol{\Lambda})$ can be interpreted as follows. The first term is standard: it counts the poor individuals among the old (surviving) population, and multiplies this by the transformed income gap. But the second term is less standard: it measures poverty among the individuals who did not survive, their fictitious incomes being assigned to them through the matrix $\boldsymbol{\Sigma}$.

As this is discussed in Lefèbvre et al (2013), various candidates are possible for the matrix $\boldsymbol{\Sigma}$. One can, for instance, use the identity matrix as the matrix $\boldsymbol{\Sigma}$. This amounts to assign to each prematurely dead person a fictitious income that is exactly equal to the income enjoyed when being alive. Such an adjustments amounts to treat premature death as something "neutral".

Another possibility is to assign, to all prematurely dead persons, a fictitious income level that is inferior to the actual income enjoyed when being alive, in such a way as to reflect the fact that a premature death is a major cause of deprivation on its own. Under that alternative adjustment, poverty is not restricted to low incomes, but also includes premature death, in line with Sen (1998)'s emphasis on the necessity to count premature death as a component of poverty. For that purpose, one possibility, which is discussed in Lefèbvre et al (2013), consists of taking the "welfare-neutral" income $y_{N}$, which brings indifference, at the individual level, between, on the one hand, survival with that income, and, on the other hand, death. That welfare neutral income is defined such a way that:

$$
U\left(u\left(y_{i}\right), u\left(y_{N}\right)\right)=U\left(u\left(y_{i}\right), \omega\right)
$$

where $U\left(u\left(y_{i}\right), u\left(y_{N}\right)\right)$ is a separable lifetime welfare function, whereas $\omega$ is the utility of being dead, usually normalized to zero. In that case, the matrix $\boldsymbol{\Sigma}$ is the column matrix at the particular "welfare-neutral" income level. Clearly, in this case, the adjusted poverty measure is different from what would have prevailed in the absence of any income-differentiated mortality; it counts a premature death as something that is a source of poverty, and, hence, is not neutral.

Adjusted FGT poverty measures tend, by construction, to take into account not only the observable poverty (i.e. of existing persons), but, also, the poverty that would have prevailed in the absence of income-differentiated mortality. In the light of this, it is tempting to ask whether or not the adjusted FGT poverty measure can bring some solution to the Mortality Paradox. In particular, under which conditions can adjusted FGT poverty measures satisfy the RMC property? Proposition 3 states that whether the adjusted FGT measure satisfies RMC or not depends on the matrices $\boldsymbol{\Sigma}$ and $\boldsymbol{\Lambda}$.

Proposition 3 The adjusted FGT measure $\hat{P}_{\alpha, \Sigma}$ does not satisfy $R M C$, except for those profiles $(\mathbf{y}, \mathbf{n}, \boldsymbol{\pi}, \boldsymbol{\Lambda})$ where the matrix $\boldsymbol{\Lambda}$ (by accident) coincides with the matrix $\mathbf{\Sigma}$. 
Proposition 3 suggests that, in general, that is, for any matrix $\boldsymbol{\Sigma}$ fixed $a$ priori as a part of the measurement instrument, the adjusted FGT poverty measure does not satisfy RMC. There is only a special case where RMC holds: when the object to be measured, i.e. $(\mathbf{y}, \mathbf{n}, \boldsymbol{\pi}, \boldsymbol{\Lambda})$, is such that the matrix $\boldsymbol{\Lambda}$ is, by accident, equal to the matrix $\boldsymbol{\Sigma}$.

Proposition 3 seems, at first glance, to provide a quite negative result. It states that adding the "missing" poor, i.e. the prematurely dead persons, does not, in general, suffice to make old-age poverty measures robust to variations in survival conditions. Whether such a robustness will be achieved or not depends on the matrix $\boldsymbol{\Sigma}$, that is, the income mobility matrix in terms of fictitious income, and on the matrix $\boldsymbol{\Lambda}$, that is, the income mobility matrix conditionally on survival. RMC requires that the matrices $\boldsymbol{\Lambda}$ and $\boldsymbol{\Sigma}$ are equal. Hence, Proposition 3 states that, if one takes the measurement instrument $\hat{P}_{\alpha, \Sigma}$ as given, it is only by accident, that is, for particular objects $(\mathbf{y}, \mathbf{n}, \boldsymbol{\pi}, \boldsymbol{\Lambda})$ to be measured, that the matrices $\boldsymbol{\Lambda}$ and $\boldsymbol{\Sigma}$ are equal, and that RMC can hold.

Note, however, that, in concrete measurement applications, there is no reason why the measurement instrument $\hat{P}_{\alpha, \Sigma}$ should be taken as given once and for all (i.e. for any measurement exercise). Clearly, instead of expecting the special case where the matrix $\boldsymbol{\Lambda}$ (which is a part of the object to be measured) is equal to the a priori fixed matrix $\boldsymbol{\Sigma}$ (which is a part of the measurement instrument), one can, when facing an object $(\mathbf{y}, \mathbf{n}, \boldsymbol{\pi}, \boldsymbol{\Lambda})$ to be measured, parametrize the matrix $\boldsymbol{\Sigma}$ in such a way that it is equal to the prevailing $\boldsymbol{\Lambda}$ matrix. Once we admit, for concrete practical measurement issues, that the matrix $\boldsymbol{\Sigma}$ can be fitted so as to be equal to the matrix $\boldsymbol{\Lambda}$, Proposition 3 admits another reading: it is possible, by parametrizing the adjusted FGT measure in a particular way, to make it robust to variations in survival conditions.

Clearly, when the object to be measured $(\mathbf{y}, \mathbf{n}, \boldsymbol{\pi}, \boldsymbol{\Lambda})$ changes, the satisfaction of RMC requires the adjusted FGT measure to be adapted as well, through a reparametrization of the matrix $\boldsymbol{\Sigma}$ in such a way as to make it equal to the matrix $\boldsymbol{\Lambda}$. The intuition behind that reparametrization goes as follows: it is only when the precise way in which fictitious incomes are assigned to prematurely dead persons coincides with what would have been assigned to those persons in case of survival that the effect of differentiated mortality on old-age poverty measurement is neutralized. Hence, if the pure income mobility process changes, RMC requires that the assignment of fictitious incomes also changes.

At this stage, it should be stressed that, although fixing $\boldsymbol{\Sigma}=\boldsymbol{\Lambda}$ guarantees that the adjusted poverty measure satisfies Robustness to Mortality Changes, there is no obvious reason why adjusted poverty measures should necessarily satisfy RMC. Actually, there exist lots of reasons for adopting a matrix $\boldsymbol{\Sigma}$ that is distinct from the matrix $\boldsymbol{\Lambda}$. Indeed, RMC implies that mortality is neutral for poverty measurement. However, one may, like Sen (1998), believe that premature death is not neutral at all for poverty measurement, but is rather a component of the poverty phenomenon to be measured. Hence, in that case, fixing $\boldsymbol{\Sigma}=\boldsymbol{\Lambda}$ is by no means desirable, and one can instead assume that the $\boldsymbol{\Sigma}$ matrix makes adjusted FGT measures rise when survival conditions deteriorate.

If the $\boldsymbol{\Sigma}$ matrix differs from the $\boldsymbol{\Lambda}$ matrix, the adjusted FGT measure does not satisfy RMC, and thus is not robust to variations in survival conditions. 
Actually, when a survival rate $\pi_{j}$ with $1 \leq j \leq K$ decreases to $\pi_{j}^{\prime}$, we have: ${ }^{11}$

$$
\begin{aligned}
\hat{P}_{\alpha, \Sigma}(\mathbf{y}, \mathbf{n}, \boldsymbol{\pi}, \boldsymbol{\Lambda}) & \gtrless \hat{P}_{\alpha, \Sigma}\left(\mathbf{y}, \mathbf{n}, \boldsymbol{\pi}^{\prime}, \boldsymbol{\Lambda}\right) \\
\operatorname{iff} \sum_{l=1}^{P-1} \lambda_{j l}\left[\frac{y_{P}-y_{l}}{y_{P}}\right]^{\alpha} & \gtrless \sum_{l=1}^{P-1} \sigma_{j l}\left[\frac{y_{P}-y_{l}}{y_{P}}\right]^{\alpha}
\end{aligned}
$$

This condition tells us how the adjusted FGT measure varies with changes in survival conditions when the matrix $\boldsymbol{\Sigma}$ differs from the matrix $\boldsymbol{\Lambda}$. If, for instance, one uses the identity matrix as the $\boldsymbol{\Sigma}$ matrix, it follows that $\sigma_{h i}=0$ except when $h=i$ (where we have $\sigma_{i i}=1$ ), with the consequence that the above condition, under $y_{j}<y_{P}$, becomes: $\sum_{l=1}^{P-1} \lambda_{j l}\left[\frac{y_{P}-y_{l}}{y_{P}}\right]^{\alpha} \gtrless\left[\frac{y_{P}-y_{j}}{y_{P}}\right]^{\alpha}$. Therefore, in the case of headcount ratio (i.e. $\alpha=0$ ), it follows that $\hat{P}_{\alpha, \Sigma}(\mathbf{y}, \mathbf{n}, \boldsymbol{\pi}, \boldsymbol{\Lambda})<$ $\hat{P}_{\alpha, \Sigma}\left(\mathbf{y}, \mathbf{n}, \boldsymbol{\pi}^{\prime}, \boldsymbol{\Lambda}\right)$. Note, however, that under $y_{j} \geq y_{P}$, the condition would become $\sum_{l=1}^{P-1} \lambda_{j l}\left[\frac{y_{P}-y_{l}}{y_{P}}\right]^{\alpha} \gtrless 0$, implying that $\hat{P}_{\alpha, \Sigma}(\mathbf{y}, \mathbf{n}, \boldsymbol{\pi}, \boldsymbol{\Lambda})>\hat{P}_{\alpha, \Sigma}\left(\mathbf{y}, \mathbf{n}, \boldsymbol{\pi}^{\prime}, \boldsymbol{\Lambda}\right)$ whatever $\alpha$ is. Hence whether the deterioration of survival conditions concerns initially poor or non-poor persons determines the direction of change when $\boldsymbol{\Sigma}$ is the identity matrix.

If, alternatively, one used, as $\boldsymbol{\Sigma}$, a matrix equal to a column matrix at the particular "welfare-neutral" income level $y_{N}$ (see above), the above condition would become: $\sum_{l=1}^{P-1} \lambda_{j l}\left[\frac{y_{P}-y_{l}}{y_{P}}\right]^{\alpha} \gtrless\left[\frac{y_{P}-y_{N}}{y_{P}}\right]^{\alpha}$. Under a very low $y_{N}$, we thus have $\hat{P}_{\alpha, \Sigma}(\mathbf{y}, \mathbf{n}, \boldsymbol{\pi}, \boldsymbol{\Lambda})<\hat{P}_{\alpha, \Sigma}\left(\mathbf{y}, \mathbf{n}, \boldsymbol{\pi}^{\prime}, \boldsymbol{\Lambda}\right)$, so that the adjusted FGT measure value is increased by the rise in mortality. Note that this is true independently from the initial income level of the prematurely dead, unlike when $\boldsymbol{\Sigma}$ is the identity matrix.

In the light of all this, it follows that the extension of lifetime income profiles of the prematurely dead persons can, in theory, lead to a more or less large rise in the measured poverty, to an extent that varies with several factors (the shape of matrix $\boldsymbol{\Sigma}$, the degree of poverty aversion $\alpha$, the income distribution under study). The goal of the next section is to use data from 11 European countries to examine the impact of those determinants on the gap between unadjusted and adjusted FGT measures of poverty at the old age.

\section{Evidence: old-age poverty in Europe}

This section proposes to illustrate, on the basis of data on old-age poverty in 11 European countries, the above theoretical discussion, by exploring how the measured old-age poverty in Europe varies with the treatment of the prematurely dead within poverty measures. For that purpose, we compare standard FGT measures (with various degrees of poverty aversion $\alpha$ ) of old-age poverty with adjusted FGT measures (under various matrixes $\boldsymbol{\Sigma}$ ) across different countries.

\section{$5.1 \quad$ Data}

The analysis is based on poverty data from the European household survey EUSILC for the year 2007, and on the life expectancy by education level made

\footnotetext{
${ }^{11}$ See the Proof of Proposition 3.
} 
available by Eurostat (2010). ${ }^{12}$ Due to the limited availability of comparable life expectancy statistics by educational level, 11 countries are included in the data set: Czech Republic, Denmark, Estonia, Finland, Hungary, Italy, Norway, Poland, Portugal, Slovenia and Sweden.

Given that the measurement interference induced by income-differentiated mortality is likely to be larger at higher ages, we will focus, throughout this section, on the measurement of old-age poverty, defined as poverty in the population aged 60 or more. The raw data on poverty in Europe in 2007 are presented in Table 1. Focusing first on simple headcount poverty measures, we see that the measured poverty at the old age varies strongly across countries. For instance, whereas only $5.5 \%$ of the population aged 60 and more is below the poverty threshold in the Czech Republic, fractions as high as one fourth of the population aged 60 and more are below the poverty line in countries such as Estonia and Portugal. Note also that, although women exhibit, in all countries under study, higher headcount poverty measures than men, the distribution of poverty across genders varies significantly across countries. In some countries, such as Hungary or Poland, the proportion of persons in poverty at the old age is approximately the same for men and women. On the contrary, in countries like the Czech Republic or Norway, the poverty gap between women and men is much larger: the prevalence of poverty at the old age is, in those countries, about three times larger among women than among men.

\begin{tabular}{c|ccc|ccc|ccc}
\hline \hline & \multicolumn{3}{|c|}{$P_{0}(\%)$} & \multicolumn{3}{c|}{$P_{1}(\%)$} & \multicolumn{3}{c}{$P_{2}(\%)$} \\
\hline Countries & total & males & females & total & males & females & total & males & females \\
\hline Czech Rep. & 5.5 & 2.6 & 7.6 & 0.7 & 0.4 & 0.9 & 0.1 & 0.1 & 0.2 \\
Denmark & 13.0 & 11.2 & 14.5 & 1.6 & 1.1 & 2.0 & 0.5 & 0.3 & 0.6 \\
Estonia & 24.5 & 15.8 & 29.2 & 4.1 & 3.1 & 4.6 & 1.3 & 1.2 & 1.3 \\
Finland & 19.1 & 14.9 & 22.2 & 2.7 & 2.1 & 3.2 & 0.7 & 0.5 & 0.7 \\
Hungary & 10.7 & 8.9 & 11.8 & 2.5 & 2.4 & 2.5 & 1.2 & 1.3 & 1.1 \\
Italy & 20.8 & 17.6 & 23.3 & 5.1 & 4.2 & 5.7 & 2.3 & 1.9 & 2.6 \\
Norway & 14.3 & 7.1 & 20.0 & 2.2 & 1.4 & 2.9 & 0.7 & 0.6 & 0.8 \\
Poland & 9.1 & 8.3 & 9.6 & 1.8 & 1.8 & 1.8 & 0.6 & 0.7 & 0.6 \\
Portugal & 24.9 & 23.1 & 26.3 & 6.4 & 5.9 & 6.9 & 2.7 & 2.5 & 2.8 \\
Slovenia & 18.1 & 11.7 & 22.5 & 4.1 & 2.9 & 5.0 & 1.6 & 1.3 & 1.8 \\
Sweden & 10.2 & 6.7 & 13.1 & 1.8 & 1.1 & 2.4 & 0.6 & 0.4 & 0.7 \\
\hline \hline
\end{tabular}

Table 1: FGT poverty measures at age $60+$, for year $2007 .{ }^{13}$

If one now considers the extent of poverty, measured through the average income gap $\left(P_{1}\right)$ or squared income gap $\left(P_{2}\right)$, further observations can be made. A first observation is that the ranking of countries in terms of poverty varies significantly according to the FGT measure used. For instance, Norway exhibits a higher proportion of poor persons in the population in comparison to Hungary $(14.3 \%$ against $10.7 \%)$, but the extent of poverty, as measured by the average income gap, is lower in Norway than in Hungary (2.2 \% against $2.5 \%) .{ }^{14}$ Furthermore, the size and sign of the poverty gender gap also varies with the FGT

\footnotetext{
${ }^{12}$ See: http://epp.eurostat.ec.europa.eu/portal/population/data/database.

${ }^{13}$ For each country, the poverty threshold is fixed at $60 \%$ of the median income (within the population that is alive).

${ }^{14} P_{1}$ measures can be interpreted as follows: individuals whose income is below the poverty line in e.g. Estonia have, on average, an income that is equal to $100-4.1=95.9 \%$ of the
} 
measure used. For instance, poverty among men is lower than among women in Poland when using headcount ratios, but the extent of poverty is larger among men than among women when focusing on the squared income gap.

Table 1 provides a contrasted picture of old-age poverty in Europe: oldage poverty levels vary across countries and gender, and are also sensitive to the FGT measure that is used. Note, however, that the picture provided by Table 1 may actually hide even larger discrepancies across European economies, which are related to differentials in survival conditions across those countries. Differential income-specific survival conditions across countries may, by leading to a more or less large number of "missing poor" - and a more or less large "hidden poverty" across those countries, distort the picture provided by Table 1. Those distortions due to different interferences caused by income-differentiated mortality may concern the different FGT measures, to various extent.

In order to identify the impact of income-differentiated mortality on poverty measurement, we need data on survival conditions by income levels. There is, to our knowledge, no lifetable by income for the European countries. However, Eurostat, produces comparable information on mortality by education. ${ }^{15}$ To illustrate the differentials in life expectancy between and within countries, Tables 2 and 3 show, respectively, the life expectancy statistics at age 60 by gender, and by education level (primary, secondary and tertiary). ${ }^{16}$

\begin{tabular}{c|ccc}
\hline \hline & \multicolumn{3}{|c}{ Life expectancy at 60} \\
\hline Countries & total & men & woman \\
\hline Czech Rep & 20.8 & 18.5 & 22.7 \\
Denmark & 21.9 & 20.3 & 23.3 \\
Estonia & 19.7 & 15.9 & 22.5 \\
Finland & 23.3 & 20.8 & 25.6 \\
Hungary & 19.4 & 16.6 & 21.7 \\
Italy & 24.3 & 22.0 & 26.2 \\
Norway & 23.4 & 21.5 & 25.1 \\
Poland & 20.8 & 17.9 & 23.2 \\
Portugal & 23.1 & 21.0 & 25.1 \\
Slovenia & 22.2 & 19.4 & 24.5 \\
Sweden & 23.6 & 22.0 & 25.1 \\
\hline \hline
\end{tabular}

Table 2: $\overline{\overline{\text { Life expectancy at age } 60 \text { by gend }}}$ er 2007.

poverty line. Regarding $P_{2}$ measures, these can be interpreted as follows. Persons whose income is below the poverty line in e.g. Estonia have, on average, an income whose relative gap with respect to the poverty line raised to the power 2 is equal to $1.3 \%$.

${ }^{15}$ See: http://epp.eurostat.ec.europa.eu/portal/population/data/database.

${ }^{16}$ Note that the data for Poland and Portugal are for year 2008 and 2009 respectively. 


\begin{tabular}{c|ccc}
\hline \hline & \multicolumn{3}{|c}{ Life expectancy at 60} \\
\hline Countries & primary & secondary & tertiary \\
\hline Czech Rep & 20.68 & 20.38 & 23.55 \\
Denmark & 21.25 & 22.02 & 22.94 \\
Estonia & 16.53 & 20.25 & 22.76 \\
Finland & 22.91 & 23.45 & 24.30 \\
Hungary & 17.91 & 21.25 & 21.20 \\
Italy & 23.87 & 25.94 & 25.82 \\
Norway & 22.57 & 23.58 & 24.43 \\
Poland & 20.28 & 20.74 & 22.74 \\
Portugal & 23.06 & 23.58 & 24.04 \\
Slovenia & 21.86 & 22.13 & 23.43 \\
Sweden & 23.06 & 23.69 & 24.58 \\
\hline
\end{tabular}

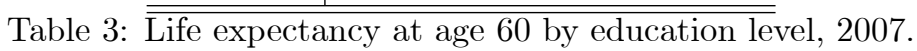

Table 2 shows the existence of significant inequalities in longevity across Europe. The lowest life expectancy at age 60 is measured in Hungary (19.4 years), while the largest one is measured in Italy (24.3 years). Table 2 also highlights that the gender gap between women and men varies across countries, from 3 years in Denmark to 6.6 years in Estonia. However, as shown in Table 3, aggregate life expectancy statistics hide also large inequalities within countries, depending on the education level. The education gap in terms of life expectancy is very small is some countries, such as Sweden, where the life expectancy at age 60 for individuals with tertiary education is only 1.5 year larger than the one for individuals with primary education only. On the contrary, the education gap is much larger in Estonia, where it is equal to about 6.2 years.

The varying life expectancy gap across countries suggests that the interferences in poverty measurement caused by income-differentiated mortality are likely to be varying across countries. In order to have a confirmation of that conjecture, we need first to use the education-specific lifetables provided by Eurostat in order to extrapolate lifetables by income levels. For that purpose, we use a weighted ordinary least square regression, in line with Bossuyt et al (2004) and Van Oyen et al (2005). Taking into account the high correlation that exists between education and income, we can extrapolate mortality by income class on the basis of the mortality by education, by relating the distributions of individuals on both dimensions. For that purpose, we first transform the absolute educational status into a relative educational status. Indeed among cohorts, the size of educational groups has changed. Young people studied more than older ones. For a given cohort, we represent each category of education by its size in the population. We then order these categories from the lowest level to the highest on a scale from 0 to $100 \%$. That is each category of income represents a percentage of the total population of the cohort. This scale gives us a distribution of the cohort population according to education. We assume that the reference of an education category is determined by its relative position, defined as the mid-point of the proportion of the category represented on the ordered scale of $100 \%$ (Pamuk, 1985, 1988). ${ }^{17}$ We then regress the life expectancy by

\footnotetext{
${ }^{17}$ For example, if the first category is given by those with at most a primary degree and represents $10 \%$ of the cohort, the mid-point reference will be $5 \%$. If the second category represents, let's say those with a secondary degree, $20 \%$ of the population, the bounds of the category in the distribution are 10 and $30 \%$ and the mid-point is $20 \%$.
} 
education on the reference mid-point of the education category by weighting for the prevalence of the category, i.e. the relative size of the educational level. The slope of the regression line represents the difference in mortality between the bottom and the top of the education hierarchy. Once estimated, the coefficients can be used to compute lifetables according to income. This is done by assuming that the social hierarchy given by the income is similar to the one given by education. We can thus apply the coefficient of one education category to the corresponding categories of income.

Figures 1 and 2 show, for the 11 European countries under study, the estimated life expectancy at age 60 by income class, for males and females respectively. For each country, life expectancy at age 60 is increasing with the income class considered. However, the longevity differential related to income inequality varies strongly across countries. The life expectancy differential is especially large in Estonia and in the Czech Republic. On the contrary, it is much lower in Sweden and Denmark. Moreover, the life expectancy gap tends to be larger for males (Figure 1) than for females (Figure 2). The significant variation in the size of the life expectancy gap in terms of income levels across countries and across gender implies that the selection bias in poverty measurement resulting from income-differentiated mortality is likely to vary also across countries and gender.

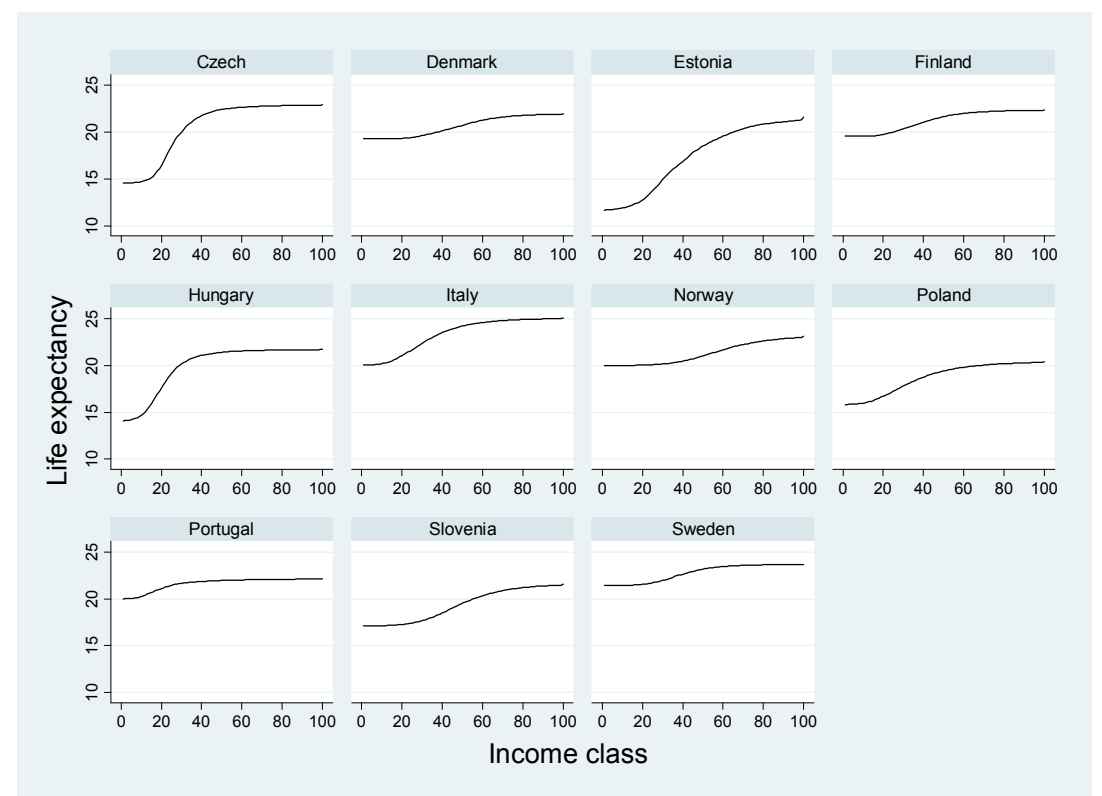

Figure 1: Life expectancy at age 60 by income class in Europe, males. 


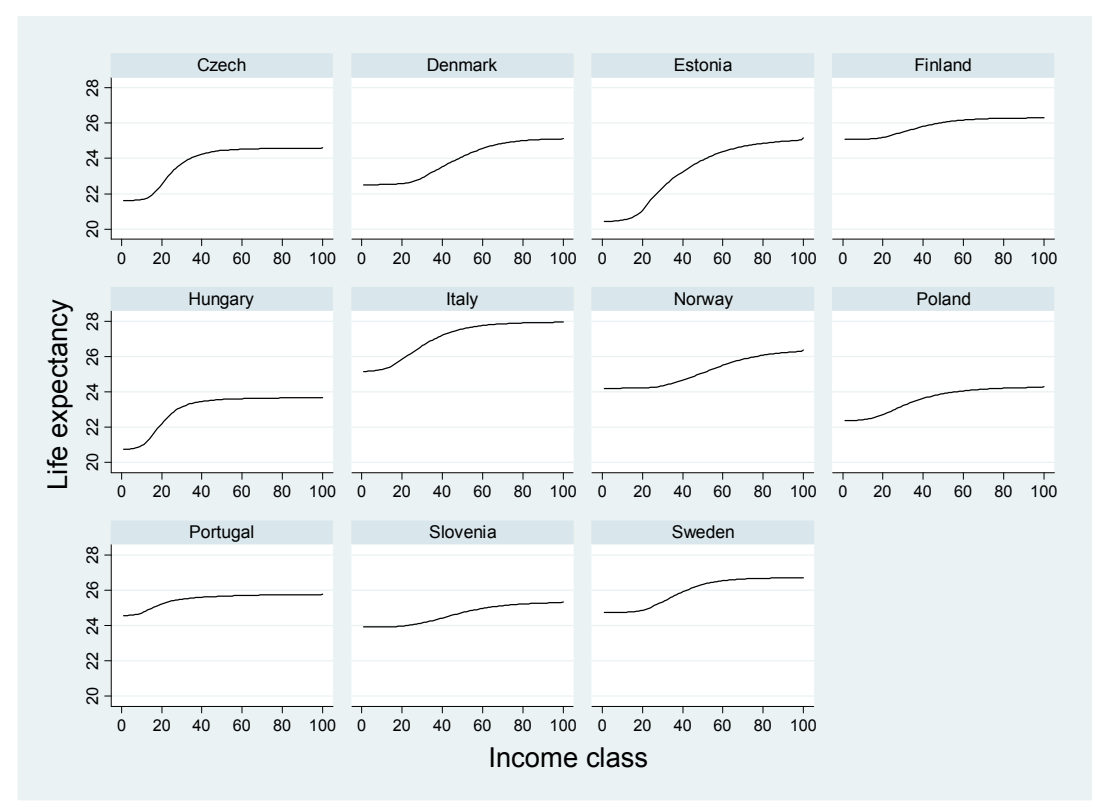

Figure 2: Life expectancy at age 60 by income class in Europe, females.

\subsection{The adjustment technique}

The adjustment of FGT poverty measures is made in two steps. First, we need to compute the number of "missing" persons for each country and each gender. Second, we need to assign a fictitious income to those "missing persons".

Regarding the first task, the number of "missing" individuals in each income class is computed, for each country and each gender, by calculating the hypothetical number of individuals of that class who would have survived if they had benefited from the survival conditions of the highest income class, for that country and that gender. Assuming a stable demography, that number of "missing" individuals in an income class can be obtained by multiplying the number of surviving individuals in that class by a coefficient equal to the ratio of incomespecific life expectancies of the highest income class to the actual income class. As an illustration of that adjustment, Table 4 shows, for each country and each gender, the life expectancy statistics at age 60 for the bottom income class and for the top income class, as well as the corresponding adjustment coefficient. 


\begin{tabular}{ccccccc}
\hline \hline & \multicolumn{2}{c}{ bottom income } & \multicolumn{2}{c}{ top income } & \multicolumn{2}{c}{ Adjustment factor } \\
\hline Countries & males & females & males & females & males & females \\
\hline Czech Rep. & 14.6 & 21.6 & 22.9 & 24.6 & 1.57 & 1.14 \\
Denmark & 19.3 & 22.5 & 22.0 & 25.1 & 1.14 & 1.12 \\
Estonia & 11.7 & 20.4 & 21.6 & 25.1 & 1.85 & 1.23 \\
Finland & 19.6 & 25.1 & 22.4 & 26.3 & 1.14 & 1.05 \\
Hungary & 14.1 & 20.8 & 21.8 & 23.7 & 1.54 & 1.14 \\
Italy & 20.0 & 25.2 & 25.1 & 28.0 & 1.25 & 1.11 \\
Norway & 20.0 & 24.2 & 23.1 & 26.4 & 1.16 & 1.09 \\
Poland & 15.8 & 22.4 & 20.4 & 24.3 & 1.29 & 1.09 \\
Portugal & 20.0 & 24.6 & 22.1 & 25.8 & 1.11 & 1.05 \\
Slovenia & 17.1 & 23.9 & 21.6 & 25.3 & 1.26 & 1.06 \\
Sweden & 21.4 & 24.7 & 23.7 & 26.7 & 1.11 & 1.08 \\
\hline \hline
\end{tabular}

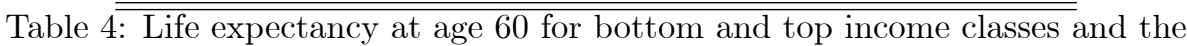
associated adjustment coefficient, 2007.

Adjustment factors for the lowest income class are larger for males than for females, in line with the higher gaps in terms of life expectancy by income class. There is also a variation in adjustment factors across countries: these are large in Estonia and Czech Republic, but much smaller for Finland and Portugal.

Regarding the second task, which consists of assigning a fictitious income to all those "missing" persons, we will, adopt two alternative approaches, which consists of two distinct matrices $\boldsymbol{\Sigma} .{ }^{18}$ The first approach consists of assigning, to each missing person, a fictitious income equal to the income previously enjoyed. That approach consists of assuming that $\boldsymbol{\Sigma}$ is an identity matrix. In that case, a premature death is, in some sense, treated as neutral for poverty measurement. Another, alternative approach, consists of counting a premature death as a source of deprivation, which leads to assign, as a fictitious income, the income equivalent to death, that is, $y_{N}$.

That welfare-neutral income, which makes an agent indifferent between, on the one hand, further life with that income, and, on the other hand, death, can be calibrated by following the work by Becker et al (2005). Taking income as a proxy for consumption, and assuming that individuals have time-additive preferences with a temporal utility function of the form $u(y)=\frac{y^{1-1 / \gamma}}{1-1 / \gamma}+\delta$, it is possible to derive the welfare-neutral income $y_{N} . y_{N}$ makes the utility associated to a life-period equal to the utility of being dead:

$$
\frac{y_{N}^{1-1 / \gamma}}{1-1 / \gamma}+\delta=0
$$

Following Becker et al (2005), we take $\gamma=1.25$. Regarding the calibration of $\delta$, we also follow Becker et al (2005), who use the estimation of $\varepsilon \equiv \frac{u^{\prime}(y) y}{u(y)}=$ 0.346 from Murphy and Topel (2003) to extrapolate the level of $\delta$. The value of $\varepsilon$ is estimated from compensating differentials for occupational mortality risks; it captures how individuals make trade-off between more income and more risk. Then, for each country, we calculate the level of $\delta$ on the basis of the average income, while assuming $\gamma=1.25$ and $\varepsilon=0.346$. Then, in a last stage, we compute, for each country, and on the basis of the parameters $\delta$ and $\gamma$ (the

\footnotetext{
${ }^{18} \mathrm{On}$ the relative strengths and weaknesses of those approaches, see Lefèbvre et al (2013).
} 
former being country-specific), the level of the welfare-neutral income $y_{N}$. Table 5 shows the values of the welfare-neutral income $y_{N}$ for each country. ${ }^{19}$

\begin{tabular}{c|c}
\hline \hline & Welfare-neutral fictitious income \\
\hline Countries & (euros 2007) \\
\hline Czech Rep & 77 \\
Denmark & 449 \\
Estonia & 54.5 \\
Finland & 347 \\
Hungary & 67 \\
Italy & 265 \\
Norway & 626 \\
Poland & 53 \\
Portugal & 136 \\
Slovenia & 149 \\
Sweden & 339 \\
\hline
\end{tabular}

Table 5: Welfare-neutral income in Europe, 2007.

The welfare-neutral income $y_{N}$ is extremely low, which is not surprising. Moreover, it varies strongly across countries, because of differences in standards of living (i.e. the level of the average income in the country), which lead to different levels of the intercept $\alpha$. Those differences may seem, at first glance, surprising. However, similar inequalities would be obtained under alternative calibration techniques using country-specific income and risk-taking attitudes. ${ }^{20}$

\subsection{Results}

Table 6 shows the adjusted FGT measures for poverty at age 60 and more obtained while assigning to each missing individual a fictitious income equal to the past income enjoyed. ${ }^{21}$ In comparison with the unadjusted FGT poverty measures (Table 1), adjusted FGT measures are significantly higher. Those higher levels reflect the inclusion, within all income classes, of the missing, prematurely dead, persons. Given that low income classes are also characterized by worse survival conditions - and thus require the addition of a larger number of missing persons -, low income classes include, proportionally, higher numbers of added people than high income classes. Note, however, that, in theory, there was no obvious reason why FGT measures would necessarily be increased by the adjustment: this depends, in fine, on whether the prematurely dead would have, in case of survival, suffered from a more severe poverty than the average surviving population. Table 6 , when compared to Table 1, shows that adjusted poverty measures are unambiguously higher than unadjusted poverty measures.

\footnotetext{
${ }^{19}$ Those figures are expressed in yearly terms.

${ }^{20}$ See, for instance, the meta-analysis made by Miller (2000) showing large differentials in the value of a statistical life across countries, depending on the income level.

${ }^{21}$ Throughout this section, the poverty line is assumed to keep the same level as before the adjustment. That assumption is in line with the framework developed in Sections 2-4. Note that this assumption constitutes an obvious simplification, since the addition of prematurely dead persons to the population may potentially affect the level of the poverty line, and, hence, poverty measures. That effect is discussed in Lefèbvre et al (2013).
} 


\begin{tabular}{c|ccc|ccc|ccc}
\hline \hline & \multicolumn{3}{|c|}{$\hat{P}_{0}(\%)$} & \multicolumn{3}{c|}{$\hat{P}_{1}(\%)$} & \multicolumn{3}{c}{$\hat{P}_{2}(\%)$} \\
\hline Countries & total & males & females & total & males & females & total & males & females \\
\hline Czech Rep. & 5.7 & 3.1 & 8.0 & 0.7 & 0.4 & 0.9 & 0.2 & 0.1 & 0.2 \\
Denmark & 13.5 & 11.6 & 15.0 & 1.7 & 1.2 & 2.1 & 0.5 & 0.3 & 0.6 \\
Estonia & 26.2 & 19.3 & 31.0 & 4.5 & 4.0 & 4.9 & 1.5 & 1.5 & 1.4 \\
Finland & 19.5 & 15.7 & 22.5 & 2.8 & 2.2 & 3.3 & 0.7 & 0.6 & 0.8 \\
Hungary & 11.7 & 10.8 & 12.4 & 2.8 & 3.0 & 2.6 & 1.3 & 1.6 & 1.2 \\
Italy & 21.9 & 19.2 & 24.1 & 5.4 & 4.6 & 6.0 & 2.4 & 2.1 & 2.7 \\
Norway & 14.7 & 7.6 & 20.5 & 2.3 & 1.5 & 3.0 & 0.7 & 0.6 & 0.8 \\
Poland & 9.6 & 9.3 & 9.9 & 1.9 & 2.0 & 1.9 & 0.7 & 0.8 & 0.6 \\
Portugal & 25.5 & 23.9 & 26.7 & 6.6 & 6.1 & 7.0 & 2.8 & 2.6 & 2.9 \\
Slovenia & 18.6 & 12.9 & 22.9 & 4.3 & 3.2 & 5.1 & 1.6 & 1.5 & 1.9 \\
Sweden & 10.4 & 7.0 & 13.4 & 1.8 & 1.1 & 2.4 & 0.6 & 0.4 & 0.7 \\
\hline \hline
\end{tabular}

Table 6: Adjusted FGT poverty measures at age $60+$, for year 2007

(fictitious income $=$ past income).$^{22}$

Note that the extent of the adjustment varies significantly across countries. The adjustment is very small in Sweden $(+0.2 \%$ for the headcount ratio), in Czech republic $(+0.2 \%)$, in Norway $(+0.4 \%)$ and in Finland $(+0.4 \%)$, but is much larger in countries such as Estonia $(+1.7 \%$ for the headcount ratio), Italy $(+1.1 \%)$ and Hungary $(+1.0 \%)$. This result reflects that the size of interferences induced by income-differentiated mortality on poverty measures vary across countries.

An interesting point to notice is that the adjustment of poverty measures can, in some cases, lead to an inversion of ranking of countries in terms of old-age poverty. For instance, whereas Portugal exhibits a higher unadjusted poverty rate at the old age than Estonia (24.9\% versus $24.5 \%$ ), this is no longer the case once poverty rates are adjusted: after adjustment, old-age poverty is lower in Portugal than in Estonia (25.5 \% against $26.2 \%$ ). Thus accounting for income-differentiated mortality affects the international comparison of old-age poverty.

Another observation concerns the gender poverty gap. Table 6 suggests that, once poverty measures are adjusted, the gap between poverty prevalences among men and women is significantly reduced. For instance, whereas the gender gap in Estonia was equal to $29.2 \%-15.8 \%=13.4 \%$ in unadjusted terms (headcount), it is reduced to $31 \%-19.3 \%=11.7 \%$ once poverty measures are adjusted. Hence the inclusion of the "missing persons" does not only affect the overall poverty prevalence, but also lowers the gender poverty gap, even though women remain, on average, more subject to poverty.

In order to quantify the effect of extending lifetime income profiles of the prematurely dead on poverty measurement at the old age, we will use the following index, which measures the differential between, on the one hand, the poverty phenomenon as measured under standard FGT measures, and, on the other hand, the poverty phenomenon as measured under adjusted FGT measures:

$$
G_{\alpha \Sigma}=1-\frac{\bar{P}_{\alpha}(\mathbf{y}, \mathbf{n}, \boldsymbol{\pi}, \boldsymbol{\Lambda})}{\hat{P}_{\alpha, \Sigma}(\mathbf{y}, \mathbf{n}, \boldsymbol{\pi}, \boldsymbol{\Lambda})}
$$

\footnotetext{
${ }^{22}$ For each country, the poverty threshold is fixed at $60 \%$ of the median income.
} 
Throughout the rest of this section, we refer to $G_{\alpha \Sigma}$ as to the "gap index".

Figure 3 shows the levels of the gap index under $\alpha$ equal to 0,1 and 2 (total population). The size of the gap index varies strongly across countries. Whereas it remains below $5 \%$ in Denmark, Finland, Norway, Portugal, Slovenia and Sweden, the gap index reaches much higher levels in Estonia, Hungary and Poland. Hence the effect of extending lifetime income profiles of the prematurely dead persons on old-age poverty measurement varies strongly across countries.

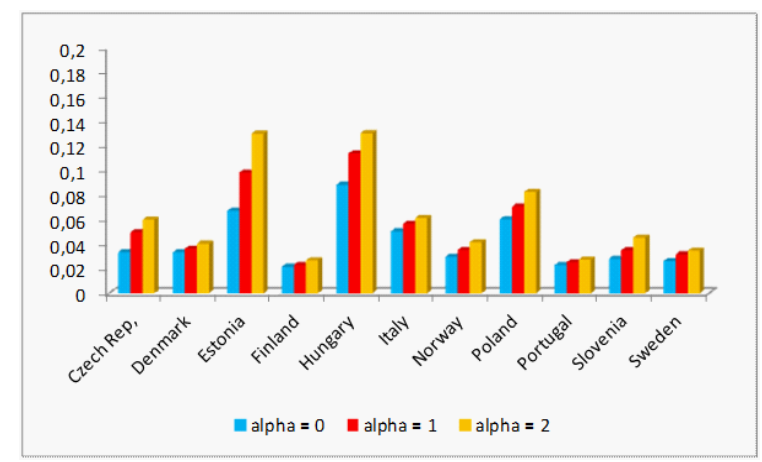

Figure 3: Gap index for FGT measures, total population (fictitious income $=$ past income)

Another important lesson from Figure 3 concerns the variation of the gap index across FGT measures of poverty for a given country. Figure 3 shows that, for the countries under study, the gap index tends to be higher for squared income gap measures $(\alpha=2)$ than for average income gap measures $(\alpha=1)$ and for headcount measures $(\alpha=0)$. Hence, the extension of income profiles of the prematurely dead leads to larger adjustments when the degree of poverty aversion is larger. Note that the extent to which the gap index increases with $\alpha$ varies across countries. Those variations reflect the differentials between income distributions across countries.

Let us now contrast those results with what is obtained under alternative fictitious incomes. For that purpose, Table 7 shows the adjusted FGT poverty measures when the fictitious income used for the extension of income profiles of the prematurely dead persons consists of the welfare-neutral income $y_{N}$. Note that the income gap or the squared income gap is expected to be more sensitive to the level of fictitious incomes than the headcount. The reason is that adopting the number 0 for all the poor made to survive or their past income that is below the poverty line has, by construction, the same impact on the headcount, but not on the income gap.

In the light of Table 7, several observations can be made. Firstly, the adjusted FGT poverty measures take, under that alternative fictitious income, much larger levels than when fictitious incomes are equalized to past incomes. That result comes from the low levels of the welfare-neutral income $y_{N}$ (see Table 5). Hence, when one considers premature death as a source of poverty and deprivation, and include it in poverty measures under the form of the income equivalent to death, poverty measures become much larger.

A second important point to be stressed concerns the strong differentials across countries. The adjustment using the welfare-neutral income as a ficti- 
tious income increases the old-age poverty rate (headcount) by $3.3 \%$ in Denmark and by $4.1 \%$ in Sweden (in comparison to the unadjusted poverty rate), but by $14 \%$ for Estonia, and by $13.5 \%$ in Czech Republic, and by $10.5 \%$ in Hungary. Those large adjustments reflect the stronger differentials in life expectancy across income classes in those countries (Figures 1 and 2).

\begin{tabular}{c|ccc|ccc|ccc}
\hline \hline & \multicolumn{3}{|c|}{$\hat{P}_{0}(\%)$} & \multicolumn{3}{c|}{$\hat{P}_{1}(\%)$} & \multicolumn{3}{c}{$\hat{P}_{2}(\%)$} \\
\hline Countries & total & males & females & total & males & females & total & males & females \\
\hline Czech Rep. & 19.0 & 24.3 & 14.4 & 14.5 & 22.0 & 7.9 & 13.7 & 21.2 & 7.1 \\
Denmark & 16.3 & 14.9 & 17.5 & 5.2 & 5.2 & 5.3 & 4.0 & 4.2 & 3.9 \\
Estonia & 38.5 & 40.1 & 37.4 & 21.5 & 30.5 & 15.3 & 18.7 & 28.4 & 12.1 \\
Finland & 23.0 & 21.2 & 24.4 & 7.3 & 9.1 & 5.9 & 5.2 & 7.4 & 3.4 \\
Hungary & 21.2 & 25.8 & 17.9 & 13.6 & 20.0 & 9.1 & 12.2 & 18.6 & 7.6 \\
Italy & 27.1 & 26.6 & 27.5 & 12.4 & 14.3 & 10.8 & 9.6 & 12.0 & 7.6 \\
Norway & 19.4 & 14.0 & 23.9 & 8.0 & 8.5 & 7.6 & 6.3 & 7.4 & 5.4 \\
Poland & 15.0 & 17.7 & 13.2 & 8.1 & 11.6 & 5.6 & 6.8 & 10.3 & 4.3 \\
Portugal & 27.8 & 27.2 & 28.3 & 9.9 & 10.7 & 9.3 & 6.2 & 7.4 & 5.3 \\
Slovenia & 23.6 & 21.8 & 25.1 & 10.5 & 14.0 & 8.0 & 7.9 & 12.1 & 4.9 \\
Sweden & 14.3 & 11.4 & 16.8 & 6.2 & 5.9 & 6.5 & 4.9 & 5.1 & 4.8 \\
\hline \hline
\end{tabular}

Table 7: Adjusted FGT poverty measures at age $60+$, for year 2007

(fictitious income $=$ welfare-neutral income).$^{23}$

A third observation concerns the size of the gender gap. Once the welfareneutral income is used to extend the income profiles of the prematurely dead, the form of the poverty gender gap is strongly altered in some countries. In Czech Republic, Estonia, Hungary and Poland, the headcount poverty measure is larger among men than among women, whereas the opposite was prevailing in unadjusted poverty measures. The reversal of the poverty gender gap observed in those countries is due to the fact that income-related differentials in survival conditions are much larger among men than among women in those countries. Therefore, once the "missing" persons are added and are assigned $y_{N}$ as a fictitious income, the poverty gender gap is reversed. Note that, in other countries, females remain, after the adjustment, more subject to poverty than men.

Finally, let us compute the gap index under that alternative adjustment of FGT poverty measures (Figure 4). The comparison of Figures 3 and 4 suggests that the effect of extending income profiles of the prematurely dead on oldage poverty measurement is much larger when using $y_{N}$ as a fictitious income in comparison to the adjustment based on past incomes. This reflects that the adjustment of old-age poverty measures is much larger once one expects a poverty measure to take into account not only the "missing poor" (as on Figure 3 ), but, also, the "missing poverty" (premature death being counted as a source of poverty).

\footnotetext{
${ }^{23}$ For each country, the poverty threshold is fixed at $60 \%$ of the median income.
} 


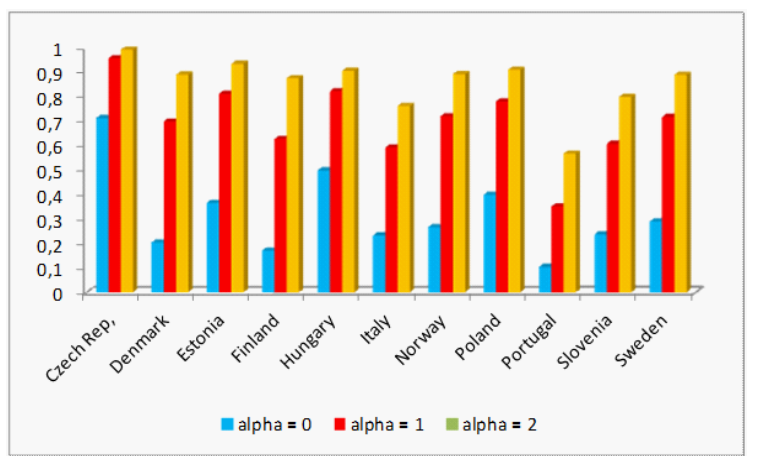

Figure 4: Gap index for FGT measures, total population (fictitious income $=$ welfare-neutral income)

Another important thing to be stressed is that, on Figure 4, the size of the gap index varies strongly across FGT poverty measures, and is much larger for squared poverty gap measures $(\alpha=2)$ than for average poverty gaps $(\alpha=1)$ and headcount ratios $(\alpha=0)$. The size of the rise of the gap index when $\alpha$ is increased is substantial, especially for countries such as Denmark, Finland, Norway, and Sweden. The intuition behind those larger increase in the gap index for those countries lies in the fact that the intensity of poverty in unadjusted terms is very low in those countries. Hence, given that unadjusted average poverty gap and squared poverty gap measures are low, the inclusion, within the income distribution, of the prematurely dead persons with very low incomes (equal to $\left.y_{N}\right)$ generates a quite strong rise in the intensity of poverty, in comparison to a low intensity in unadjusted terms. That rise is reinforced by the fact that poverty lines are much larger in those countries. Those larger poverty threshold lead to a higher intensity of poverty when the "missing persons" are added with a fictitious income equal to the welfare-neutral income (which is much lower than the poverty line).

In the light of Figures 3 and 4, it appears that the effects of extending income profiles of the prematurely dead on old-age poverty measurement vary strongly across classes of FGT measures, and across countries. Eastern economies are characterized by larger income-related differentials in survival conditions. Therefore, the adjustment strongly raises headcount poverty measures for those countries. On the contrary, Nordic economies suffer from lower income-related differentials in survival conditions, so that the number of "missing" persons is lower. This explains why Nordic economies exhibit lower gap indexes when $\alpha=0$. However, for Nordic countries, the adjustment has a bigger impact on distribution-sensitive poverty indicators $(\alpha>0)$, since these were very low in unadjusted terms, and since the poverty line is larger in Nordic economies. Hence, once we take the intensity of poverty into account, Nordic countries exhibit larger adjustments that are close to the ones of Eastern economies.

In sum, the effect of extending lifetime income profiles of the prematurely dead on old-age poverty measurement varies depending on: (1) the fictitious incomes assigned to the prematurely dead persons; (2) the postulated degree of poverty aversion $\alpha$; (3) the shape of the (unadjusted) income distribution; (4) 
the strength of the income/mortality relationship. The determinant (1) plays a crucial role: when the fictitious income assigned to the prematurely dead persons is equal to the welfare-neutral income, adjusted poverty measures are much larger than unadjusted poverty measures. But even for a given adjustment technique, there exist significant variations in the adjustment depending on the degree of poverty aversion $\alpha(2)$ : distribution-sensitive measures lead to larger adjustments than headcount measures. Factors (3) and (4) are well illustrated by international comparisons. International differentials in the size of adjustment - in particular the opposition between Nordic and Eastern Europe mirror both international differentials in income-related survival conditions and in the income distribution (including the level of the poverty line).

\section{Concluding remarks}

By mechanically reducing the proportion of poor persons in the population, income-differentiated mortality introduces some noise in the measurement of poverty. This leads to the Mortality Paradox: a deterioration of the survival conditions faced by the poor can generate a decline in the measured poverty. That reduction is puzzling, and is a mere consequence of the absence of the "missing poor" in the population on which poverty is measured, and of the ignorance of premature death as a major aspect of poverty (Sen 1998).

This paper examined whether this puzzle for poverty measurement affects FGT poverty measures. Are FGT measures subject to the Mortality Paradox? If yes, are all subclasses of FGT measures equally subject to the Mortality Paradox, whatever the degree of poverty aversion is?

To answer those questions, we developed a model of income mobility with risky lifetime to study how robust FGT measures are to variations in survival conditions. We showed that FGT old-age poverty measures do not, in general, satisfy the Robustness to Mortality Changes condition (RMC). Actually, it is only under some particular income mobility process - where all individuals face the same expected extent of poverty in case of survival to the old age - that FGT measure satisfy RMC.

Under general conditions, FGT measures do not satisfy RMC. This motivated us to propose an adjustment of FGT measures, by extending the lifetime income profiles of the prematurely dead, in line with Kanbur and Mukherjee (2007). Then, we identified conditions under which so-adjusted FGT measures do satisfy the RMC property. Actually, adjusted FGT measures satisfy RMC when the assignment of fictitious income to the prematurely dead is made similar to the pure income mobility process conditional on survival.

Finally, we showed, on the basis of data on old-age poverty in 11 European economies (2007), that the measured extent of old-age poverty varies strongly, depending on the particular treatment of the prematurely dead, that is, depending on how the "missing poor" are taken into account (or not) in the poverty measure. The effect of extending the lifetime income profiles varies with the fictitious incomes assigned to the prematurely dead, and, also, with the degree of aversion to poverty within FGT measures. The adjustment is lower for headcount measures than for measures taking the intensity of poverty into account. The size of the adjustment varies also across countries, depending on the shape of the income distribution, and on the severity of overmortality due to low in- 
come. Whereas Eastern European countries exhibit much larger adjustments than Nordic European countries under headcount measures, both Eastern and Nordic countries exhibit large adjustments when the intensity of poverty is also taken into account.

All in all, our study illustrates that the interferences caused by incomedifferentiated mortality constitute a general problem for poverty measurement. Economies with large (unadjusted) poverty rates and strong overmortality for the poor are concerned by the Mortality Paradox. But more surprisingly, richer economies with little income-differentiated mortality are also subject to it. The reason is that, in their case, taking the "missing poor" and the "hidden poverty" into account creates a much bigger contrast with the standards of the surviving populations. Hence, even in rich economies, how one treats the prematurely dead affects the measured poverty.

\section{References}

Backlund, E., Sorlie, P. \& Johnson, N. (1999) A comparison of the relationships of education and income with mortality: the national longitudinal mortality study. Social Science and Medicine, 49 (10): 1373-1384.

Becker, G., Philipson, T.\& Soares, R. (2005) The quantity and quality of life and the evolution of world inequality. American Economic Review, 95 (1): 277-291.

Bossert, W., Chakravarty, S.R. \& D'Ambrosio, C. (2011) Poverty and time. Journal of Economic Inequality, 10 (2): 145-162.

Bossuyt, N., Gadeyne, S., Deboosere, P. \&Van Oyen, H. (2004) Socio-economic inequalities in healthy expectancy in Belgium. Public Health 118:3-10

Deaton, A. \& Paxson, C. (1998) Aging and inequality in income and health. American Economic Review, 88: 248-253.

Deaton, A. (2003) Health, inequality and economic development. Journal of Economic Literature, 41: 113-158.

Duggan, J., Gillingham, R. \& Greenlees, J. (2007) Mortality and lifetime income: evidence from U.S. Social Security Records. IMF Working Paper, 07/15.

Duleep, H.O. (1986) Measuring the effect of income on adult mortality using longitudinal administrative record data. Journal of Human Resources, 21 (2): 238-251.

Dutta, I., Roope, L. \& Zank, H. (2013). On intertemporal poverty measures: the role of affluence and want. Social Choice and Welfare, 41 (4): 741-762.

Foster, J., Greer, J. \& Thorbecke, E. (1984) A class of decomposable poverty measures. Econometrica, 52 (3): 761-766.

Foster, J. (2009) A class of chronic poverty measures. In: Addison, T., Hulme, D., Kanbur, R. (eds.) Poverty Dynamics: Interdisciplinary Perspectives, 59-76. Oxford University Press, Oxford.

Hoy, M. \& Zheng, B. (2011) Measuring Lifetime Poverty. Journal of Economic Theory, 146 (6): 2544-2562.

Jusot, F. (2004) Mortalité et revenu en France: construction et résultats d'une enquête cas-témoins. Santé, Société et Solidarité, 3 (2): 173-186.

Kanbur, R. \& Mukherjee, D. (2007) Premature mortality and poverty measurement. Bulletin of Economic Research, 59 (4): 339-359.

Lefebvre, M., Pestieau, P. \& Ponthiere, G. (2013) Measuring Poverty Without the Mortality Paradox. Social Choice and Welfare, 40 (1): 285-316. 
Miller, T. (2000) Variations between countries in values of statistical life. Journal of Transport Economics and Policy 34 (2): 169-188.

Murphy, K. \& Topel, R. (2003) The Economic value of medical research. In: K. Murphy and R. Topel (eds.): Measuring the Gains from Medical Research: An Economic Approach. The University of Chicago press.

Pamuk E.R. (1985) Social class inequality in mortality from 1921 to 1972 in England and Wales. Population Studies, 39:17-31

Pamuk E.R. (1988) Social class inequality in infant mortality in England and Wales from 1921 to 1980. European Journal of Population 4:1-21

Salm, M. (2007) The effect of pensions on longevity: evidence from Union Army veterans. IZA Discussion Paper 2668.

Sen A.K. (1998) Mortality as an indicator of economic success and failure. Economic Journal 108:1-25

Snyder, S. \& W. Evans (2006) The effect of income on mortality: evidence from the social security notch. Review of Economics and Statistics, 88 (3): 482-495.

Van Oyen H., Bossuyt N., Deboosere P., Gadeyne S., Abatith E., \& Demarest S. (2005) Differential inequity in health expectancy by region in Belgium. Social Prevention Medicine 50(5): $301-310$

\section{Appendix}

\subsection{Proof of Proposition 1}

The FGT poverty measure at the old age is equal to:

$$
\bar{P}_{\alpha}(\mathbf{y}, \mathbf{n}, \boldsymbol{\pi}, \boldsymbol{\Lambda})=\frac{1}{\sum_{k=1}^{K} \sum_{i=1}^{K} \pi_{i} n_{i} \lambda_{i k}} \sum_{k=1}^{P-1} \sum_{i=1}^{K} \pi_{i} n_{i} \lambda_{i k}\left[\frac{y_{P}-y_{k}}{y_{P}}\right]^{\alpha}
$$

To show that the FGT measure does not, under an unrestricted space $\mathbf{Y} \times \mathbf{N} \times \mathbf{\Pi} \times \mathbf{L}$, satisfy RMC, let us consider a shift from $\pi_{l}$ to $\pi_{l}^{\prime}>\pi_{l}$. We have:

$$
\bar{P}_{\alpha}(\mathbf{y}, \mathbf{n}, \boldsymbol{\pi}, \boldsymbol{\Lambda})=\bar{P}_{\alpha}\left(\mathbf{y}, \mathbf{n}, \boldsymbol{\pi}^{\prime}, \mathbf{\Lambda}\right)
$$

if and only if:

$$
\begin{aligned}
& \frac{1}{\sum_{j \neq l}^{K} \pi_{j} n_{j}+\pi_{l} n_{l}}\left[\sum_{j \neq l}^{K} \pi_{j} n_{j}\left(\sum_{k=1}^{P-1} \lambda_{j k}\left[\frac{y_{P}-y_{k}}{y_{P}}\right]^{\alpha}\right)+\pi_{l} n_{l}\left(\sum_{k=1}^{P-1} \lambda_{l k}\left[\frac{y_{P}-y_{k}}{y_{P}}\right]^{\alpha}\right)\right] \\
= & \frac{1}{\sum_{j \neq l}^{K} \pi_{j} n_{j}+\pi_{l}^{\prime} n_{l}}\left[\sum_{j \neq l}^{K} \pi_{j} n_{j}\left(\sum_{k=1}^{P-1} \lambda_{j k}\left[\frac{y_{P}-y_{k}}{y_{P}}\right]^{\alpha}\right)+\pi_{l}^{\prime} n_{l}\left(\sum_{k=1}^{P-1} \lambda_{l k}\left[\frac{y_{P}-y_{k}}{y_{P}}\right]^{\alpha}\right)\right]
\end{aligned}
$$

That condition is, in general, not satisfied. In order to show this, let us consider a simple example where $\alpha=0$ and with two income levels, with $y_{1}<y_{2}$. We assume $y_{2}=y_{P}$. Assume also that the matrix $\boldsymbol{\Lambda}$ is equal to: $\left(\begin{array}{ll}1 & 0 \\ 0 & 1\end{array}\right)$, that $\pi_{1}=0.1$ and $\pi_{2}=0.9$ and that $n_{1}=n_{2}=1$. Consider a variation in $\pi_{1}$ from 0.1 to 0.2 . The measured poverty is initially equal to:

$$
\bar{P}_{\alpha}(\mathbf{y}, \mathbf{n}, \boldsymbol{\pi}, \boldsymbol{\Lambda})=\frac{1}{0.1(1)+0.9(1)}(0.1(1))=0.1
$$


whereas when $\pi_{1}=0.2$ it becomes:

$$
\bar{P}_{\alpha}\left(\mathbf{y}, \mathbf{n}, \boldsymbol{\pi}^{\prime}, \boldsymbol{\Lambda}\right)=\frac{1}{0.2(1)+0.9(1)}(0.2(1))=\frac{0.2}{1.1}>0.1
$$

That simple counterexample suffices to show that $\bar{P}_{\alpha}(\mathbf{y}, \mathbf{n}, \boldsymbol{\pi}, \boldsymbol{\Lambda})$ does not satisfy RMC.

\subsection{Proof of Proposition 2}

Let us first introduce the following notation:

$$
Z_{i} \equiv \sum_{k=1}^{P-1} \lambda_{i k}\left[\frac{y_{P}-y_{k}}{y_{P}}\right]^{\alpha}
$$

Hence, the FGT old-age poverty measure can be rewritten as:

$$
\bar{P}_{\alpha}(\mathbf{y}, \mathbf{n}, \boldsymbol{\pi}, \boldsymbol{\Lambda})=\frac{\pi_{1} n_{1} Z_{1}+\pi_{2} n_{2} Z_{2}+\ldots+\pi_{K} n_{K} Z_{K}}{\pi_{1} n_{1}+\pi_{2} n_{2}+\ldots+\pi_{K} n_{K}}
$$

The condition in Proposition 2 amounts to assume that:

$$
Z_{1}=Z_{2}=\ldots=Z_{K}=Z
$$

Hence, under that condition, we have:

$$
\bar{P}_{\alpha}(\mathbf{y}, \mathbf{n}, \boldsymbol{\pi}, \boldsymbol{\Lambda})=Z
$$

so that $\bar{P}_{\alpha}(\mathbf{y}, \mathbf{n}, \boldsymbol{\pi}, \boldsymbol{\Lambda})$ does not depend upon survival probabilities $\pi_{j}$.

\subsection{Proof of Corollary 1}

Assume now that $\alpha=0$. Then $Z_{i}$ becomes:

$$
Z_{i}=\sum_{k=1}^{P-1} \lambda_{i k}
$$

The condition stated in Corollary 1 amounts to assume, when $\alpha=0$, that:

$$
Z_{1}=Z_{2}=\ldots=Z_{K}=Z
$$

Hence, under that condition, the FGT old-age poverty measure can be written as:

$$
\bar{P}_{\alpha}(\mathbf{y}, \mathbf{n}, \boldsymbol{\pi}, \boldsymbol{\Lambda})=\frac{\pi_{1} n_{1} Z+\pi_{2} n_{2} Z+\ldots+\pi_{K} n_{K} Z}{\pi_{1} n_{1}+\pi_{2} n_{2}+\ldots+\pi_{K} n_{K}}=Z
$$

which is independent from survival probabilities. 


\subsection{Proof of Proposition 3}

The adjusted FGT poverty measure at the old age is equal to:

$$
\hat{P}_{\alpha, \Sigma}(\mathbf{y}, \mathbf{n}, \boldsymbol{\pi}, \boldsymbol{\Lambda})=\frac{1}{\sum_{j=1}^{K} n_{j}}\left[\begin{array}{l}
\sum_{j=1}^{K} \pi_{j} n_{j}\left(\sum_{k=1}^{P-1} \lambda_{j k}\left[\frac{y_{P}-y_{k}}{y_{P}}\right]^{\alpha}\right) \\
+\sum_{j=1}^{K}\left(1-\pi_{j}\right) n_{j}\left(\sum_{k=1}^{P-1} \sigma_{j k}\left[\frac{y_{P}-y_{k}}{y_{P}}\right]^{\alpha}\right)
\end{array}\right]
$$

It is easy to show that, for any matrix $\boldsymbol{\Sigma}$, the adjusted poverty measure does not satisfy RMC. For that purpose, consider a shift from $\pi_{l}$ to $\pi_{l}^{\prime}>\pi_{l}$. We have:

$$
\hat{P}_{\alpha, \Sigma}(\mathbf{y}, \mathbf{n}, \boldsymbol{\pi}, \boldsymbol{\Lambda})=\hat{P}_{\alpha, \Sigma}\left(\mathbf{y}, \mathbf{n}, \boldsymbol{\pi}^{\prime}, \boldsymbol{\Lambda}\right)
$$

if and only if:

$$
\begin{array}{r}
\frac{1}{\sum_{j=1}^{K} n_{j}}\left[\begin{array}{c}
\sum_{j \neq l}^{K} \pi_{j} n_{j}\left(\sum_{k=1}^{P-1} \lambda_{j k}\left[\frac{y_{P}-y_{k}}{y_{P}}\right]^{\alpha}\right)+\pi_{l} n_{l}\left(\sum_{k=1}^{P-1} \lambda_{l k}\left[\frac{y_{P}-y_{k}}{y_{P}}\right]^{\alpha}\right) \\
+\sum_{j \neq l}^{K}\left(1-\pi_{j}\right) n_{j}\left(\sum_{k=1}^{P-1} \sigma_{j k}\left[\frac{y_{P}-y_{k}}{y_{P}}\right]^{\alpha}\right)+\left(1-\pi_{l}\right) n_{l}\left(\sum_{k=1}^{P-1} \sigma_{l k}\left[\frac{y_{P}-y_{k}}{y_{P}}\right]^{\alpha}\right)
\end{array}\right] \\
=\frac{1}{\sum_{j=1}^{K} n_{j}}\left[\begin{array}{c}
\sum_{j \neq l}^{K} \pi_{j} n_{j}\left(\sum_{k=1}^{P-1} \lambda_{j k}\left[\frac{y_{P}-y_{k}}{y_{P}}\right]^{\alpha}\right)+\pi_{l}^{\prime} n_{l}\left(\sum_{k=1}^{P-1} \lambda_{l k}\left[\frac{y_{P}-y_{k}}{y_{P}}\right]^{\alpha}\right) \\
+\sum_{j \neq l}^{K}\left(1-\pi_{j}\right) n_{j}\left(\sum_{k=1}^{P-1} \sigma_{j k}\left[\frac{y_{P}-y_{k}}{y_{P}}\right]^{\alpha}\right)+\left(1-\pi_{l}^{\prime}\right) n_{l}\left(\sum_{k=1}^{P-1} \sigma_{l k}\left[\frac{y_{P}-y_{k}}{y_{P}}\right]^{\alpha}\right)
\end{array}\right]
\end{array}
$$

That equality can be simplified as:

$$
\begin{aligned}
& \pi_{l} n_{l}\left(\sum_{k=1}^{P-1} \lambda_{l k}\left[\frac{y_{P}-y_{k}}{y_{P}}\right]^{\alpha}\right)+\left(1-\pi_{l}\right) n_{l}\left(\sum_{k=1}^{P-1} \sigma_{l k}\left[\frac{y_{P}-y_{k}}{y_{P}}\right]^{\alpha}\right) \\
= & \pi_{l}^{\prime} n_{l}\left(\sum_{k=1}^{P-1} \lambda_{l k}\left[\frac{y_{P}-y_{k}}{y_{P}}\right]^{\alpha}\right)+\left(1-\pi_{l}^{\prime}\right) n_{l}\left(\sum_{k=1}^{P-1} \sigma_{l k}\left[\frac{y_{P}-y_{k}}{y_{P}}\right]^{\alpha}\right)
\end{aligned}
$$

or, alternatively,

$$
n_{l}\left(\sum_{k=1}^{P-1} \lambda_{l k}\left[\frac{y_{P}-y_{k}}{y_{P}}\right]^{\alpha}\right)\left(\pi_{l}-\pi_{l}^{\prime}\right)=n_{l}\left(\sum_{k=1}^{P-1} \sigma_{l k}\left[\frac{y_{P}-y_{k}}{y_{P}}\right]^{\alpha}\right)\left(\pi_{l}-\pi_{l}^{\prime}\right)
$$

This can be simplified to:

$$
\sum_{k=1}^{P-1} \lambda_{l k}\left[\frac{y_{P}-y_{k}}{y_{P}}\right]^{\alpha}=\sum_{k=1}^{P-1} \sigma_{l k}\left[\frac{y_{P}-y_{k}}{y_{P}}\right]^{\alpha}
$$

For an arbitrary matrix $\boldsymbol{\Sigma}$, that condition is, in general, not satisfied. But if the matrix $\boldsymbol{\Sigma}$ is set equal to the matrix $\boldsymbol{\Lambda}$, then the condition is satisfied, so that the measured poverty is invariant to changes in survival probabilities. 